\title{
Effects of pressure gradients on turbulent premixed flames
}

\author{
By D. Veynante ${ }^{1}$ AND T. Poinsot ${ }^{2}$
}

\section{Motivations and objectives}

Most ducted turbulent flames are subjected to external pressure gradients. Compared to "free" flames, i.e. turbulent flames without externally imposed pressure gradients, the combination of the external pressure gradients with the large density changes found in premixed flames may lead to strong modifications of the flame structure. These modifications are mainly due to the differential buoyancy effects between cold, heavy reactants and hot, light products. They affect turbulent transport along with many characteristics of the flame itself, such as the flame speed, thickness, wrinkling, and local structure. Pressure gradients are also a key mechanism for the counter-gradient turbulent transport described below. Accordingly, studying the effects of pressure gradients on premixed turbulent flames is an important issue both for fundamental understanding of turbulent combustion and for modeling.

Using the assumption of single-step chemistry, the mass fractions of the reactive species are all linearly related (Williams 1985) and may be expressed in terms of a single reduced mass fraction: the reaction progress variable $c$. The progress variable ranges from zero to unity in the fresh and fully burnt gases, respectively. Using the classical Favre decomposition, a quantity $q$ can be split into a mass-weighted mean, $\tilde{q} \equiv \overline{\rho q} / \bar{\rho}$, and a turbulent fluctuation, $q^{\prime \prime}$. The transport equation for the mean reaction progress variable $\tilde{c}$ may be written as:

$$
\frac{\partial \bar{\rho} \tilde{c}}{\partial t}+\frac{\partial \bar{\rho} \tilde{u}_{i} \tilde{c}}{\partial x_{i}}+\frac{\partial \overline{\rho u_{i}^{\prime \prime} c^{\prime \prime}}}{\partial x_{i}}=-\frac{\overline{\partial \mathcal{J}_{k}}}{\partial x_{k}}+\overline{\dot{\omega}}_{c}
$$

where $\rho$ is the mass density, $u_{i}$ is the flow velocity, $\mathcal{J}_{k}$ is the molecular diffusion flux, $\dot{\omega}_{c}$ is the volumetric production rate of the chemical reaction, and the overbar superscript denotes conventional Reynolds ensemble-averaging. Equation (1) has the form of a standard turbulent transport equation where the rate of change of $\tilde{c}$ results from a balance between convection by the mean flow, convection by the turbulent flow, molecular diffusion, and chemical reaction. The contribution of molecular diffusion is usually neglected for high Reynolds number flows. In Eq. (1), two terms need to be modeled: the mean reaction rate $\overline{\dot{\omega}_{c}}$ and the turbulent transport $\overline{\rho u_{i}^{\prime \prime} c^{\prime \prime}}$ terms. The first term has received considerable attention in recent years

1 Laboratoire EM2C, Ecole Centrale Paris, France

2 Institut de Mecanique des Fluides de Toulouse and CERFACS, France 
and various models have been derived and incorporated into practical codes for turbulent combustion. The second term, however, has received considerably less attention and is generally described with a simple classical gradient eddy-viscosity model:

$$
\overline{\rho u_{i}^{\prime \prime} c^{\prime \prime}}=\bar{\rho} \widetilde{u_{i}^{\prime \prime} c^{\prime \prime}}=-\frac{\mu_{t}}{\sigma_{c}} \frac{\partial \widetilde{c}}{\partial x_{i}}
$$

where $\mu_{t}$ denotes the turbulent dynamic viscosity and $\sigma_{c}$ a turbulent Schmidt number.

Both theoretical and experimental research (Bray et al., 1981, 1982; Shepherd et $a l ., 1982$ ) have shown the occurrence of counter-gradient transport in some turbulent flames: flames where the turbulent flux $\overline{\rho u_{i}^{\prime \prime} c^{\prime \prime}}$ and the $\widetilde{c}$ gradient, $\partial \widetilde{c} / \partial x_{i}$, have the same sign in opposition with the prediction of Eq. (2). This is generally due to the differential effect of pressure gradients on cold reactants and hot products. Recent studies based on direct numerical simulations of turbulent premixed flames without externally imposed pressure gradients (Trouvé et al.,1994; Rutland \& Cant, 1994) have confirmed that counter-gradient diffusion was found in simulations, but that classical gradient diffusion was also possible. A criterion indicating the presence of gradient or counter-gradient diffusion in atmospheric flames has been derived by Veynante et al.(1995). This criterion leads to a reduced number called $N_{B}$, or the Bray number:

$$
N_{B}=\frac{\tau}{2 \alpha\left(u^{\prime} / s_{l}^{0}\right)}
$$

where $s_{l}^{0}$ is the laminar flame speed, $u^{\prime}$ is the RMS turbulent velocity, $\tau$ is the heat release factor defined as $\tau=T_{b} / T_{u}-1$, with $T$ being the temperature and indices $u$ and $b$ referring to the fresh and burnt gases, respectively. The term $\alpha$ is an efficiency function of order unity, introduced to take into account the reduced ability of small turbulent vortices to affect the flame front. For low values of $N_{B}$, typically $N_{B} \leq 1$, where flames exist in relatively large turbulence intensity, gradient diffusion is obtained. For large values of $N_{B}$ counter-gradient diffusion occurs. In fact, counter-gradient turbulent diffusion is promoted by heat release and thermal expansion (increasing values of $\tau$ ) whereas increased turbulence intensity tends to induce gradient transport.

The work of Veynante et al.(1995) was performed for free flames without externally imposed pressure gradients or volume forces, such as gravity. Since turbulent transport in flames appears to be controlled by a dynamic balance between fresh and burnt gases, confined flames subjected to strong pressure gradients should exhibit a large sensitivity to these gradients. For example, we expect that imposing a pressure gradient on a turbulent flame exhibiting counter-gradient diffusion may lead to a gradient-diffusion situation. This change could affect the flame brush thickness, the turbulent flame speed, and finally the complete structure of the turbulent flame brush, as described in a number of papers by Masuya \& Libby, 1981; Bray et al., 1982.

Our objective in this study is to explore the effects of pressure gradients on premixed turbulent flames using direct numerical simulations. We will first recall 
the physics of this phenomenon and how pressure gradients may be included in a simulation for reacting flows in Section 2. We will also describe the most general theory to treat the problem, i.e. the Bray-Moss-Libby formulation. The simulation used for this work will be described in Section 3. Section 4 will present the structure of laminar flames submitted to pressure gradients. Section 5 will present results for turbulent flames. Finally, Section 6 will describe a model for the turbulent flux incorporating the effects of pressure gradients.

\section{Pressure gradients in premixed flames}

\subsection{The Bray-Moss-Libby approach for turbulent transport in premixed flames}

Bray et al.(1989) have proposed a simple algebraic closure for the reaction term $\overline{\dot{\omega}}_{c}$ in Eq. (1), but focus their attention on the turbulent transport term $\bar{\rho} \widetilde{u_{i}^{\prime \prime} c^{\prime \prime}}$. In the Bray-Moss-Libby (BML) approach, the flame is analyzed as a thin flame sheet, or "flamelet," separating fresh reactants $(c=0)$ and fully burnt products $(c=1)$. This assumption leads to a bimodal probability density for the progress variable $c$, and the turbulent flux is then expressed, according to Bray 1980, as:

$$
\bar{\rho} \widetilde{u_{i}^{\prime \prime} c^{\prime \prime}}=\bar{\rho} \widetilde{c}(1-\widetilde{c})\left(\overline{u_{i b}}-\overline{u_{i u}}\right)
$$

where $\overline{u_{i}}{ }_{u}$ and $\overline{u_{i b}}$ are the conditional mean velocities within the unburnt and burnt gases, respectively. The occurrence of counter-gradient transport may be easily explained from this expression. Let us consider a left-traveling flame along the $x_{i}$ direction $\left(\partial \widetilde{c} / \partial x_{i}>0\right)$. Thermal expansion and the associated flow acceleration through the flame, along with favorable buoyancy and/or pressure gradients, will tend to make $\overline{u_{i b}}$ larger than $\overline{u_{i u}}$, thereby promoting counter-gradient turbulent diffusion of $\widetilde{c}$, resulting in $\bar{\rho} u_{i}^{\prime \prime} c^{\prime \prime}>0$. Under the Bray-Moss-Libby approach, the

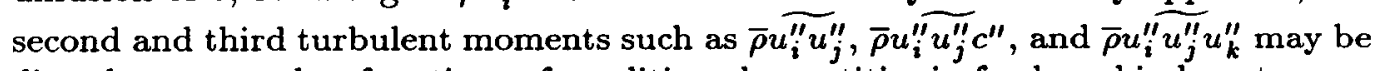
directly expressed as functions of conditioned quantities in fresh and in burnt gases. Nevertheless, conditional quantities such as $\left(\overline{u_{i b}}-\overline{u_{i u}}\right)$ are difficult to close and an alternative approach must be pursued for estimating the turbulent transport: $\bar{\rho} u_{i}^{\prime \prime} c^{\prime \prime}$. A simple algebraic closure based on the eddy viscosity concept cannot be used here. In the Bray-Moss-Libby model, closure is achieved by a transport equation for $\overline{\rho u_{i}^{\prime \prime} c^{\prime \prime}}$ (Bray 1980, Bray et al.1989, Bray 1990). A brief derivation of this equation is provided here, including a constant volume force $F_{i}^{v}$ and a constant acceleration $\Gamma_{i}$. We start from the momentum equation:

$$
\frac{\partial \rho u_{i}}{\partial t}+\frac{\partial \rho u_{i} u_{j}}{\partial x_{j}}=-\frac{\partial P}{\partial x_{i}}+F_{i}^{v}+\rho \Gamma_{i}+\frac{\partial \tau_{i k}}{\partial x_{k}}
$$

and the equation for the progress variable $c$ :

$$
\frac{\partial \rho c}{\partial t}+\frac{\partial \rho u_{i} c}{\partial x_{j}}=-\frac{\partial \mathcal{J}_{k}}{\partial x_{k}}+\dot{\omega}_{c}
$$

where $P, \tau_{i j}$ and $\mathcal{J}_{k}$ are respectively the pressure, the viscous stress tensor and the molecular diffusive flux of $c$. Multiplying Eq. (5) by $c$ and Eq. (6) by $u_{i}$, then 
adding and averaging the two resulting equations leads to a transport equation for $\bar{\rho} \widetilde{u_{i}}$. In a similar way, adding the averaged version of Eq. (5) multiplied by $\widetilde{c}$ and the averaged form of Eq. (6) multiplied by $\tilde{u_{i}}$ leads to a transport equation for $\bar{\rho} \tilde{u_{i}} \tilde{c}$. Subtracting the two resulting equations provides a transport equation for the turbulent flux $\bar{\rho} u_{i}^{\prime \prime} c^{\prime \prime}$ :

$$
\begin{aligned}
& \frac{\partial}{\partial t} \bar{\rho} \widetilde{u_{i}^{\prime \prime} c^{\prime \prime}}+\frac{\partial}{\partial x_{j}}\left(\bar{\rho} \widetilde{U_{j}} \widetilde{u_{i}^{\prime \prime} c^{\prime \prime}}\right)=-\frac{\partial}{\partial x_{j}}\left(\bar{\rho} u_{j}^{\prime \prime} u_{i}^{\prime \prime} c^{\prime \prime}\right)-\bar{\rho} \widetilde{u_{i}^{\prime \prime} u_{j}^{\prime \prime}} \frac{\partial \widetilde{c}}{\partial x_{j}}-\bar{\rho} \widetilde{u_{j}^{\prime \prime} c^{\prime \prime}} \frac{\partial \widetilde{U_{i}}}{\partial x_{j}} \\
& -\overline{c^{\prime \prime}} \frac{\partial \bar{p}}{\partial x_{i}}-\overline{c^{\prime \prime} \frac{\partial p^{\prime}}{\partial x_{i}}}-\overline{u_{i}^{\prime \prime} \frac{\partial \mathcal{J}_{k}}{\partial x_{k}}}+\overline{c^{\prime \prime} \frac{\partial \tau_{i k}}{\partial x_{k}}}+\bar{\rho} \overline{u_{i}^{\prime \prime} \dot{\omega}}+\overline{c^{\prime \prime}} F_{i}^{v}
\end{aligned}
$$

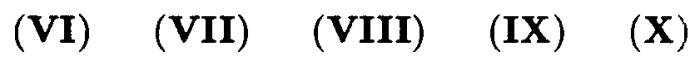

Bray et al.(1981) studied each term in Eq. (7) and proposed some approximations. For example, they explored the role of the mean pressure gradient term (VI) assuming that this term is so large that only cross dissipation terms (VIII and IX) can provide a balance, leading to a turbulent flux directly proportional to the pressure gradient. All terms in Eq. (7) may be extracted from our direct numerical simulations (Trouvé et al., 1994; Veynante et al., 1995) to validate these analyses.

Two main comments arise from Eq. (7). First, the mean pressure gradient appears explicitly in the source term (VI) $\left(\overline{c^{\prime \prime}} \partial \bar{p} / \partial x_{i}\right)$. Under the BML analysis, $\overline{c^{\prime \prime}}$ may be easily closed (Masuya \& Libby, 1981):

$$
\overline{c^{\prime \prime}}=\bar{c}-\widetilde{c}=\tau \frac{\widetilde{c}(1-\widetilde{c})}{1+\tau \widetilde{c}}
$$

which is exact for an infinitely thin flame front. This quantity, being always positive, indicates a pressure decrease from fresh to burnt gases and tends to promote countergradient diffusion, or positive values of the turbulent flux $\widetilde{\rho} u_{i}^{\prime \prime}$.

Another important feature concerning the present numerical simulations deals with volume and buoyancy forces. A constant volume force $F_{i}^{v}$ leads to a source term in Eq. (7) with a similar form for the pressure gradient term, whereas a constant acceleration force $\Gamma_{i}$ does not. However, the introduction of $F_{i}^{v}$ or $\Gamma_{i}$ has a direct influence on the mean pressure gradient $\partial \bar{P} / \partial x$ (term VI). So, terms (VI) and (XI) should be grouped to describe the effect of $F_{i}^{v}$ or $\Gamma_{i}$. We start from the averaged momentum transport equation:

$$
\frac{\partial \bar{\rho} \tilde{u_{i}}}{\partial t}+\frac{\partial \bar{\rho} \tilde{u_{i}} \tilde{u_{j}}}{\partial x_{j}}+\frac{\partial \bar{\rho} \widetilde{u_{i}^{\prime \prime} u_{j}^{\prime \prime}}}{\partial x_{j}}=-\frac{\partial \bar{P}}{\partial x_{i}}+F_{i}^{v}+\bar{\rho} \Gamma_{i}+\frac{\partial \bar{\tau}_{i k}}{\partial x_{k}}
$$

For sufficiently large volume and/or buoyancy forces, an equilibrium between these forces and the mean pressure gradient can be used, which leads to the hydrostatic approximation:

$$
\frac{\partial \bar{P}}{\partial x_{i}}=F_{i}^{v}+\bar{\rho} \Gamma_{i}
$$


Therefore, to first order, term (VI $+\mathrm{XI})$ becomes:

$$
-\overline{c^{\prime \prime}}\left(\frac{\partial \bar{P}}{\partial x_{i}}-F_{i}^{v}\right)=-\overline{c^{\prime \prime}} \bar{\rho} \Gamma_{i}
$$

Both $F_{i}^{v}$ and $\Gamma_{i}$ induce a mean pressure gradient in the flow field (see Eq. 5). However, only $\Gamma_{i}$ will directly affect the balance of $\bar{\rho} \widetilde{u_{i}^{\prime \prime} c^{\prime \prime}}$ (Eq. 7 ). * Accordingly, our study of the influence of the mean pressure gradient on turbulent transport will be conducted using a constant acceleration $\Gamma_{i}$.

\subsection{Physical and numerical issues related to pressure gradients in flames}

Theoretical models indicate that both normal and tangential pressure gradients influence turbulent flames. Masuya \& Libby (1981) have studied confined oblique flames and have shown that, for a given pressure gradient, turbulent transport in normal and transverse directions are correlated. As a first step, we will only consider pressure gradients in the mean propagation direction $x_{1}$ : only $F_{1}^{v}=F_{v}$ and $\Gamma_{1}=\Gamma$ may be nonzero. All pressure gradients are scaled by the pressure gradient inside the laminar flame zone given by:

$$
\left|\nabla P_{l a m}\right| \simeq \rho\left(s_{l}^{0}\right)^{2} \tau / \delta_{l}^{0}
$$

where $\delta_{l}^{0}$ is the unstrained laminar flame thickness that is obtained from the maximum temperature gradient $\delta_{l}^{0}=\operatorname{Max}\left(\frac{d T}{d x}\right) /\left(T_{b}-T_{u}\right)$.

The pressure gradient $\nabla P_{l a m}$ is created by dilatation inside the flame zone. It is large, but due to the thinnish flame front, the overall pressure jump between fresh and burnt gases remains small since $\Delta P / P \simeq \tau \gamma\left(s_{l}^{0} / c\right)^{2}$ where the ratio of flame to sound speeds is of the order of 0.001 . On the contrary, volume forces or external pressure gradients are imposed on distances much larger than the flamelet thickness and will overcome the effect of dilatation in turbulent flame brushes. These effects and their relative importance in various flames may be quantified in terms of two quantities: the reduced external pressure gradient $\nabla P_{e x t}^{+}$, and the reduced mean pressure gradient $\nabla P_{\text {mean }}^{+}$. We consider these gradients positive when the pressure increases when going from the fresh to the burnt gases. Figures 1 and 2 illustrate how these gradients may be estimated in a turbulent flame brush of thickness $l_{b}$ with and without an external pressure gradient. With no external pressure gradient, corresponding to Fig. 1, the pressure jump across the flame will be conserved but spread over the flame brush thickness $l_{b}$ so that the mean pressure gradient in the flame brush will be $\nabla P_{\text {mean }}^{+} \simeq \nabla P_{l a m}^{+} \delta_{l}^{0} / l_{b}$. In the case of an externally imposed pressure gradient, corresponding to Fig. $2, \nabla P_{\text {mean }}^{+}$will be of the order of $\nabla P_{e x t}^{+}$ everywhere in the flow; although the maximum instantaneous pressure gradient may still be found at the flame, $\nabla P_{\text {ext }}^{+}$will be dominant over the flame brush thickness.

Pressure gradients in real flames are imposed either by flame confinement, as in ducted flows, or by gravity. Typical values of $\nabla P_{l a m}^{+}, \nabla P_{e x t}^{+}$, and $\nabla P_{m e a n}^{+}$are

* This result is due to the fact that, contrary to a constant acceleration, a constant volume force does not introduce buoyancy phenomena. 


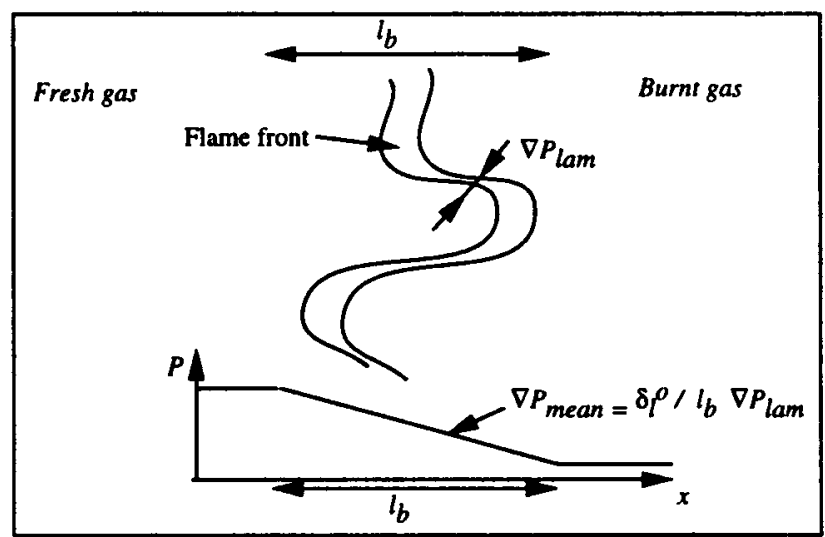

FIGURE 1. Pressure gradients in a free flame $\left(\nabla P_{e x t}^{+}=0\right)$.

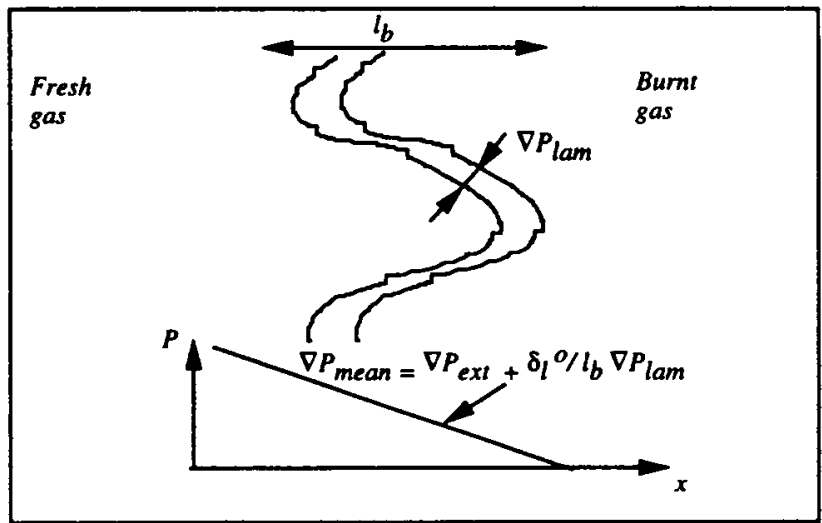

FiguRE 2. Pressure gradients in a flame with imposed pressure gradient.

given in Table I for different turbulent premixed flames along with their dimensional values. In the case of "free" flames, the pressure gradient is imposed by the flame itself. The flame data correspond to the experiment of Schefer et al.(1982). The flame brush thickness is of the order of the integral length scale $l_{t}=1 \mathrm{~cm}$.

TABLE I. Typical pressure gradients for a propane/air flame ( $\mathrm{P}=1 \mathrm{~atm}, \phi=1.2)$

\begin{tabular}{|c|c|c|c|c|c|c|c|}
\hline & $\begin{array}{l}s_{l}^{0} \\
\mathrm{~m} / \mathrm{s}\end{array}$ & $\begin{array}{l}\delta_{l}^{0} \\
\mathrm{~m}\end{array}$ & $T_{b} / T_{u}$ & $\begin{array}{l}\left|\nabla P_{l a m}\right| \\
\mathrm{Pa} / \mathrm{m}\end{array}$ & $\begin{array}{l}\left|\nabla P_{\text {ext }}\right| \\
\mathrm{Pa} / \mathrm{m}\end{array}$ & $\left|\nabla P_{e x t}^{+}\right|$ & $\left|\nabla P_{\text {mean }}^{+}\right|$ \\
\hline Free flame & 0.32 & 0.0005 & 6.5 & 250 & 0 & 0. & 0.05 \\
\hline $1 \mathrm{~g}$ flame & 0.32 & 0.0005 & 6.5 & 250 & $\simeq 10$ & 0.5 & 0.5 \\
\hline Ducted flame & 0.32 & 0.0005 & 6.5 & 250 & 1000 & 5 & 5 \\
\hline
\end{tabular}


These estimations indicate that the largest pressure gradients will be obtained in ducted flames where large pressure differences are created. Gravity alone will create smaller effects. At this point, it is worth discussing the differences between flames subjected to a constant acceleration, such as gravity, or to a constant pressure gradient. In the first case, the induced pressure gradient is $\rho \Gamma$, which is different in the fresh and burnt gases, see below. The induced pressure gradient will be constant in the latter case. For both cases, however, the pressure gradient will induce differential acceleration for fresh and burnt gas pockets in both cases thus leading to a modification of turbulent transport. Most authors therefore expect similar effects from constant acceleration or from pressure gradients.

There are at least three ways to introduce pressure gradients in a direct numerical simulation of premixed turbulent flames: impose a constant volume force $F_{v}$; impose a pressure gradient through the boundary conditions; or impose a constant acceleration $\Gamma$, i.e. a volume force that is a function of the local density $F_{g}=\rho \Gamma$, where $\Gamma=g$ in the case of gravity. All of these techniques produce an imposed pressure gradient, see Appendix I. However, we have seen that the first solution leads to a flow where the pressure gradient $\frac{\partial P}{\partial x}$ is compensated everywhere by the volume force $F_{v}$ and has no effect on $\bar{\rho} \widetilde{u^{\prime \prime} c^{\prime \prime}}$. The second solution was investigated, but it is difficult to implement in a simulation if the mean flow remains one-dimensional, which is required for statistical purposes. In this paper, we will use only the third solution with various values of the acceleration $\Gamma$.

\section{Direct numerical simulation of premixed flames with pressure gradients}

The present direct numerical simulations were performed with a two-dimensional version of NTMIX. A complete description of this code may be found in Haworth \& Poinsot (1992) or Poinsot \& Lele (1992). It solves the fully compressible NavierStokes equations with a single irreversible reaction Fuel $\rightarrow$ Products. Variable density as well as viscosity and transport coefficients are taken into account. The conservation equations solved by the simulation are:

$$
\begin{aligned}
\frac{\partial \rho}{\partial t}+\frac{\partial}{\partial x_{i}}\left(\rho u_{i}\right) & =0 \\
\frac{\partial \rho u_{i}}{\partial t}+\frac{\partial}{\partial x_{j}}\left(\rho u_{i} u_{j}\right) & =-\frac{\partial p}{\partial x_{i}}+F_{i}^{v}+\rho \Gamma_{i}+\frac{\partial \tau_{i j}}{\partial x_{j}} \\
\frac{\partial \rho E}{\partial t}+\frac{\partial}{\partial x_{i}}\left[(\rho E+p) u_{i}\right] & =\left(F_{i}^{v}+\rho \Gamma_{i}\right) u_{i}+\frac{\partial}{\partial x_{i}}\left(u_{j} \tau_{i j}\right) \\
& +\frac{\partial}{\partial x_{i}}\left(\lambda \frac{\partial T}{\partial x_{i}}\right)+Q \dot{w} \\
\frac{\partial(\rho \tilde{Y})}{\partial t}+\frac{\partial}{\partial x_{i}}\left(\rho \tilde{Y} u_{i}\right) & =\frac{\partial}{\partial x_{i}}\left(\rho \mathcal{D} \frac{\partial \tilde{Y}}{\partial x_{i}}\right)-\dot{w}
\end{aligned}
$$

where 


$$
\begin{aligned}
\rho E & =\frac{1}{2} \rho \sum_{k=1}^{3} u_{k}^{2}+\frac{p}{\gamma-1}, \\
\tau_{i j} & =\mu\left(\frac{\partial u_{i}}{\partial x_{j}}+\frac{\partial u_{j}}{\partial x_{i}}-\frac{2}{3} \delta_{i j} \frac{\partial u_{k}}{\partial x_{k}}\right), \\
\dot{w} & =\dot{w}_{R} / Y_{R}^{o}=\rho \tilde{Y} B \exp (-\beta / \alpha) \exp \left(\frac{-\beta(1-\theta)}{1-\alpha(1-\theta)}\right) .
\end{aligned}
$$

In these expressions $\rho$ is the mass density, $p$ is the thermodynarnic pressure, $\rho E$ is the total energy density, $Q$ designates the heat of reaction per unit mass of fresh mixture $\left(Q=-\Delta h_{f}^{o} Y_{R}^{o}\right.$ where $\Delta h_{f}^{o}$ is the heat of reaction per unit mass of reactant). $F_{i}^{v}$ and $\Gamma_{i}$ are the $i$-component of the constant volume force $F^{v}$ and the constant acceleration $\Gamma$, respectively. The reduced temperature is $\Theta=\left(T-T_{u}\right) /\left(T_{b}-T_{u}\right)$. The fresh gas temperature is $T_{u}$, and $T_{b}$ is the adiabatic flame temperature for unity Lewis number. The activation temperature is $T_{a} . B$ is the pre-exponential factor and the coefficients $\alpha$ and $\beta$ are the temperature factor and the reduced activation energy, respectively:

$$
\alpha=\left(T_{b}-T_{u}\right) / T_{b} ; \quad \beta=\alpha T_{a} / T_{b} .
$$

The mass fraction of the reactants $Y_{R}$ is nondimensionalized by the initial mass fraction of reactants $Y_{R}^{o}$ in the fresh gases: $\widetilde{Y}=Y_{R} / Y_{R}^{o}$. This varies from 1 in the fresh gases to 0 in the burnt gases.

We assume that the gas mixture is a perfect gas with constant molar mass and a specific heat ratio $\gamma=1.4$. The thermal conductivity $\lambda$ and the diffusion coefficient $\mathcal{D}$ are obtained from the viscosity coefficient $\mu$ according to

$$
\lambda=\mu C_{p} / P_{r} \text { and } \mathcal{D}=\mu /\left(\rho S_{\mathrm{c}}\right),
$$

where the Prandtl number $P_{r}$ and the Schmidt number $S c$ are constant. As a consequence the Lewis number $L_{e}=S_{c} / P_{r}$ is also constant. The viscosity $\mu$ is a function of temperature: $\mu=\mu_{u}\left(T / T_{u}\right)^{b}$.

The computational domain is $L_{x}$ by $L_{y}$ with $N_{x}$ by $N_{y}$ grid points. Two box sizes have been used: the small box has $N_{x}=N_{y}=257$. The aspect ratio is $L_{y} / L_{x}$ $=2.5$. The second box, or large box, has $N_{x}=257$ and $N_{y}=1025$. The aspect ratio for this box is $L_{y} / L_{x}=6.66$. The following parameters have been used for both cases:

TABLE II. Fixed parameters for direct numerical simulations of flames subjected to pressure gradients

\begin{tabular}{lllllllll}
\hline$R e=c L_{x} / \nu$ & $L e$ & $P r_{o}$ & $T_{a} / T_{b}$ & $T_{b} / T_{u}$ & $b$ & $s_{l}^{0} / c$ & $\delta_{l}^{0} / L_{x}$ & $N x$ \\
\hline 12000. & 1. & 0.75 & 8. & 4. & 0.76 & 0.0159 & 0.027 & 257
\end{tabular}

where the speed of sound and kinematic viscosity in the fresh gases are denoted by $c$ and $\nu$. 


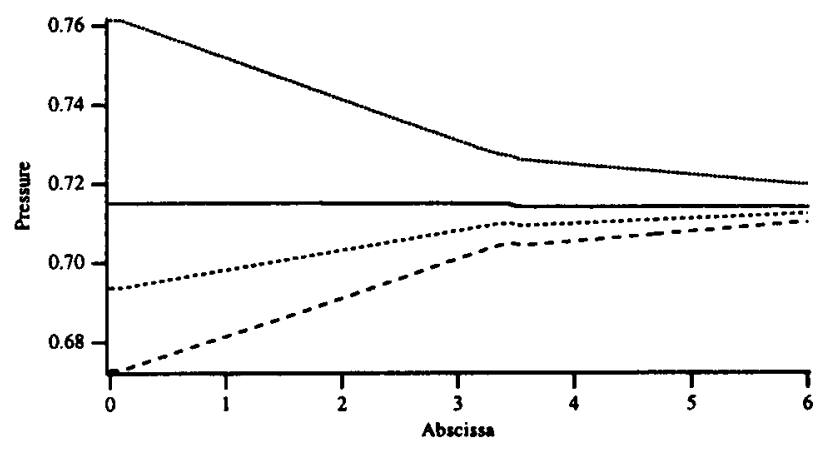

FIgURE 3. Pressure profiles in 1D laminar flames without and with imposed acceleration $g^{*} . g^{*}=0(-) ; g^{*}=-6.25(\cdots \cdots \cdots) ; g^{*}=3.12(---)$; $g^{*}=6.25(---)$

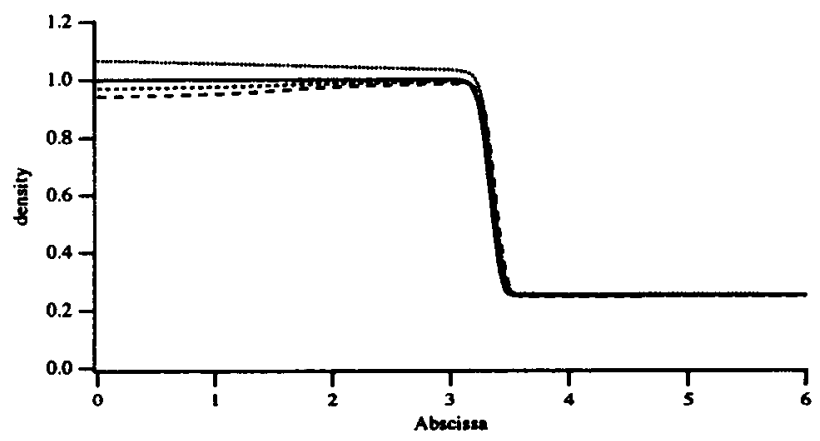

FIGURE 4. Density profiles in 1D laminar flames without and with imposed acceleration $\left.g^{*} . g^{*}=0(-) ; g^{*}=-6.25(\cdots \cdots)\right) ; g^{*}=3.12(----) ; g^{*}=6.25$ $(---)$

\section{Laminar flames submitted to pressure gradients}

First, one dimensional laminar flames are computed without and with an imposed constant acceleration $\Gamma$. Introducing the reduced acceleration, $g^{*}=\Gamma \delta_{l}^{0} /\left(s_{l}^{0}\right)^{2}$, which may be viewed as the inverse of a Froude number, four values of $g^{*}$ are considered here: $g^{*}=0$ (no imposed acceleration), $g^{*}=-6.25$ (favorable pressure gradient), $g^{*}=3.12$ and $g^{*}=6.25$ (adverse pressure gradient). Pressure profiles are plotted as a function of the downstream locations in Fig. 3 for the four $g^{*}$ values. As expected, pressure gradients are constant for each side of the flame front but decrease by a factor $T_{b} / T_{u}=4$ between fresh and burnt gases due to density changes. The pressure drop due to thermal expansion is apparent for the $g^{*}=0$ 


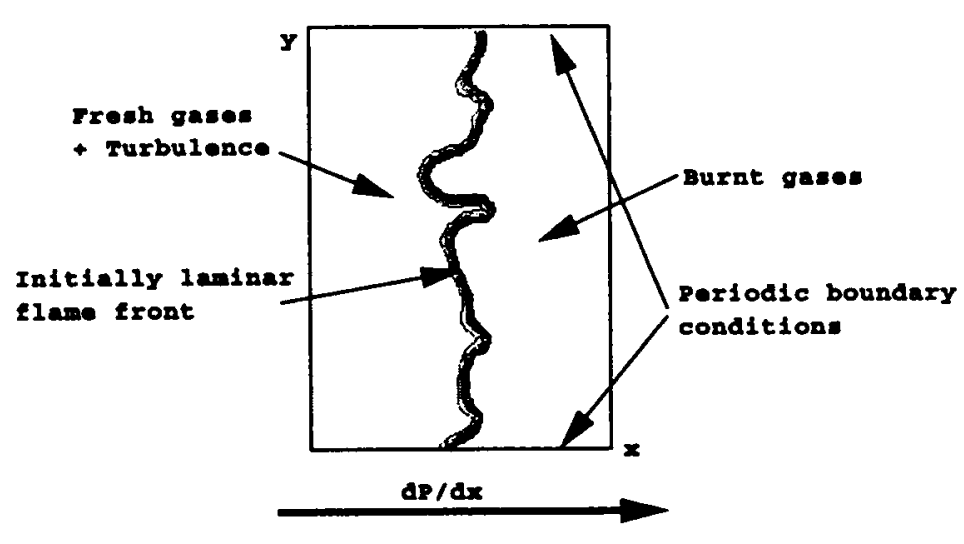

FIGURE 5. Configuration for the numerical simulations.

case.

For all of the $g^{*}$ values considered, the laminar flame structure is not affected by the imposed acceleration: flame thickness, reaction rate and mass fraction profiles remain unchanged. Nevertheless, due to the pressure gradient, a weak change in density, similar to the one described in Appendix 1, is observed inside the fresh or burnt gases, see Fig. 4, but remains negligible compared to the one induced by thermal expansion. The same trend is noticed for the flow velocity which is modified by about $3 \%$ by the pressure gradient and a factor of 4 by thermal expansion.

TABLE III. Numerical parameters for direct numerical simulations of $2 \mathrm{D}$ turbulent flames

\begin{tabular}{llllll}
\hline Case & $u_{0}^{\prime} / s_{l}^{0}$ & $l_{t} / \delta_{l}^{0}$ & $g^{*}$ & $N_{x}$ & $N_{y}$ \\
\hline $\mathrm{A}$ & 5. & 3.5 & 0. & 257 & 1025 \\
$\mathrm{~B}$ & 5. & 3.5 & -6.25 & 257 & 1025 \\
$\mathrm{C}$ & 2. & 2.7 & 0. & 257 & 257 \\
$\mathrm{D}$ & 2. & 2.7 & 3.12 & 257 & 257 \\
$\mathrm{E}$ & 2. & 2.7 & 6.25 & 257 & 257 \\
\hline
\end{tabular}

\section{Turbulent flames submitted to pressure gradients}

The previously computed 1D laminar flames are used as initial solutions for 2D flame-turbulence interaction simulations (Fig. 5). A Passot-Pouquet turbulence spectrum, with given turbulence intensity $u^{\prime}$ and integral length scale $l_{t}$, is superposed on the combustion field (Haworth \& Poinsot, 1992). Two sets of numerical 


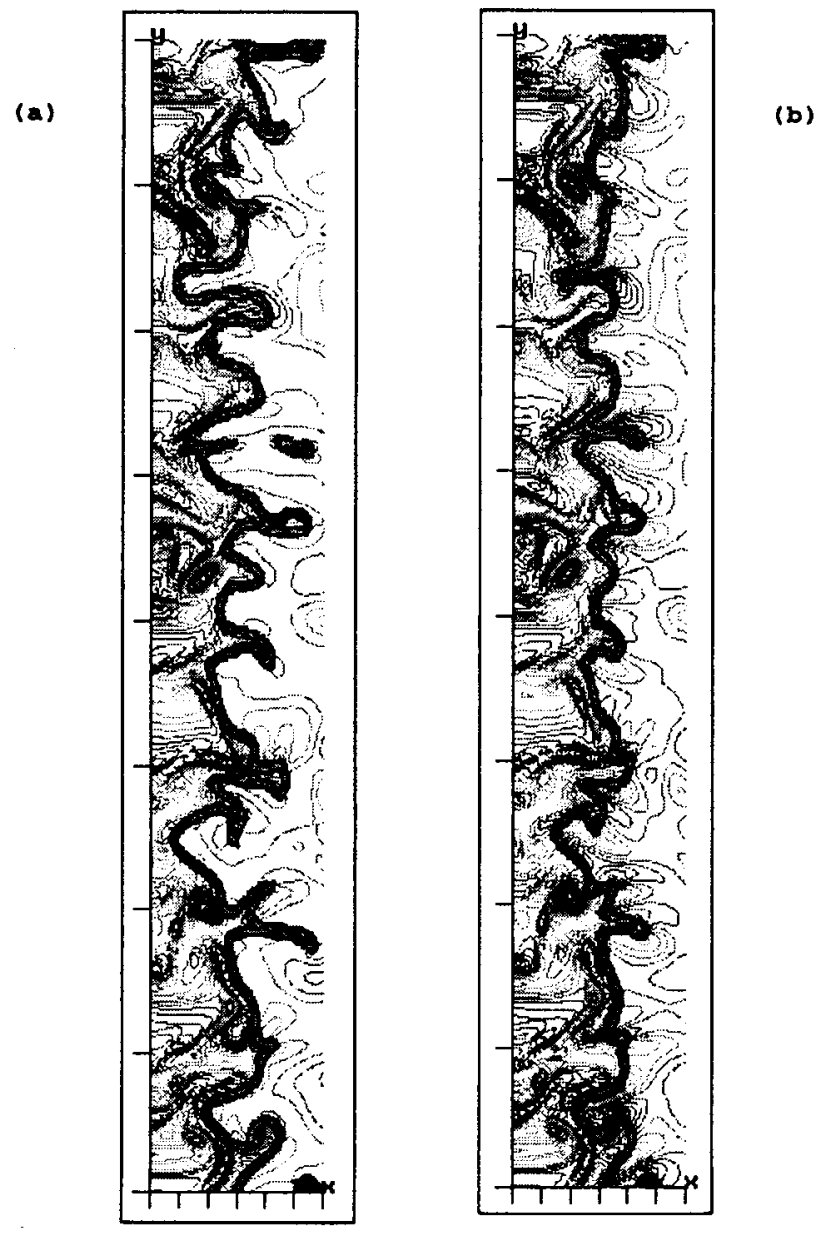

FIGURE 6. Superimposed instantaneous temperature and vorticity fields. Initial turbulence level $u_{0}^{\prime} / s_{l}^{0}=5$. (a) No imposed pressure gradient $\left(g^{*}=0\right.$. - case A); (b) Favorable imposed pressure gradient $\left(g^{*}=-6.25\right.$ - case B).

simulations have been conducted. The first set, consisting of runs A and B, starts from a high turbulence level $\left(u_{0}^{\prime} / s_{l}^{0}=5\right)$ and exhibits a classical gradient turbulence transport. The initial Bray number for this flow is $N_{B}=0.6$. A favorable pressure gradient (i.e. $\left.\nabla P_{\text {mean }}^{+}<0\right)$ is imposed with a positive acceleration $(\Gamma>0)$ to reach a counter gradient diffusion situation. The second set, consisting of runs C-E, starts from a low turbulence level $\left(u_{0}^{\prime} / s_{l}^{0}=2\right)$, counter-gradient situation, with an initial Bray number of $N_{B}=1.5$. Under an adverse pressure gradient (i.e. $\nabla P_{\text {mean }}^{+}>0$ ), the flow is found to exhibit gradient turbulent transport. Numerical parameters are displayed on Table III where $g^{*}$ is the reduced imposed acceleration 

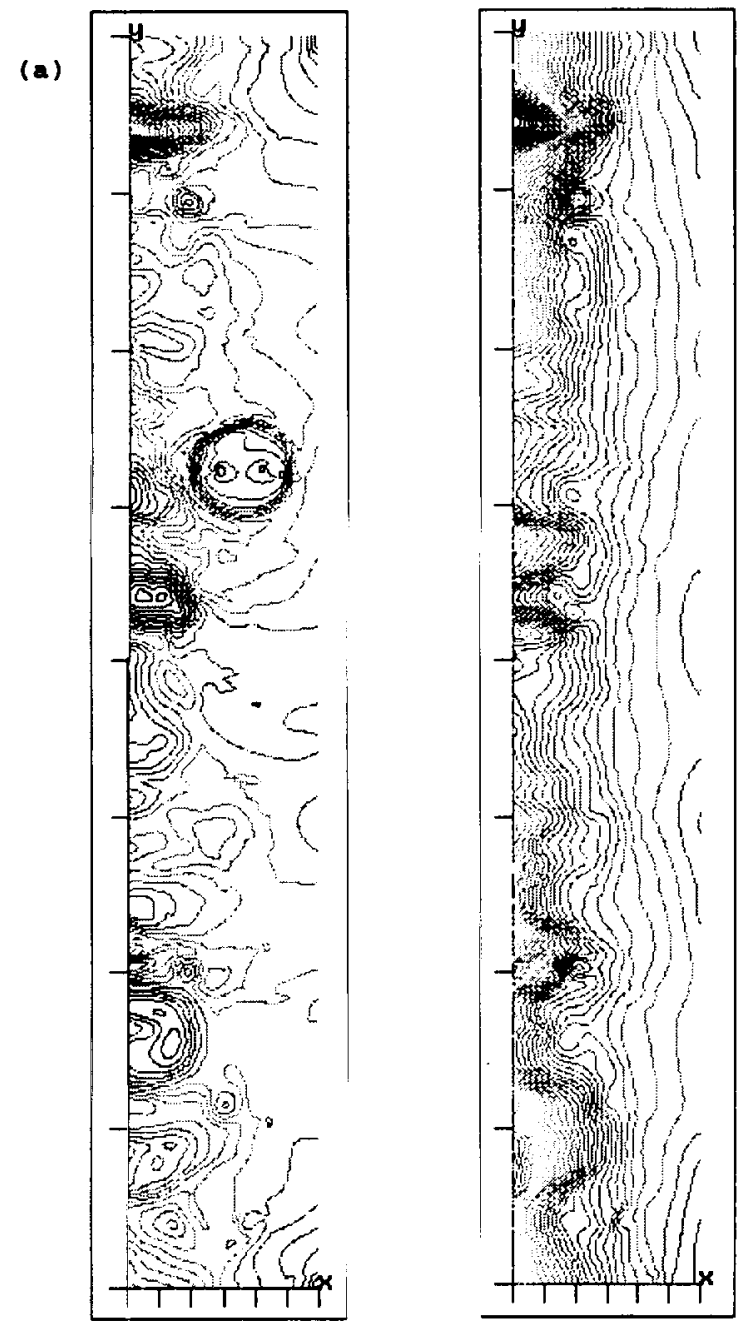

(b)

FiguRE 7. Instantaneous pressure field. Initial turbulence level $u_{0}^{\prime} / s_{l}^{0}=5$. (a) No imposed pressure gradient $\left(g^{*}=0\right.$. - case A); (b) Favorable imposed pressure gradient $\left(g^{*}=-6.25\right.$ - case B).

$\left(g^{*}=\Gamma \delta_{l}^{0} /\left(s_{l}^{0}\right)^{2}\right)$. The values of $\nabla P_{m e a n}^{+}$were chosen of the order of the pressure gradient found in the experiment of Shepherd et al.(1982).

5.1 Effect of the mean pressure gradient on the turbulent flame structure

Instantaneous temperature and vorticity fields are displayed in Fig. 6 for an initial turbulence level $u_{0}^{\prime} / s_{l}^{0}=5$ without (case A) and with (case B) an imposed mean pressure gradient. Corresponding pressure fields are displayed in Fig. 7. The flame structures are quite different. Due to the favorable pressure gradient $(\partial \bar{P} / \partial x<0)$, the wrinkling of the flame front is lower and the turbulent flame brush is thinner. 


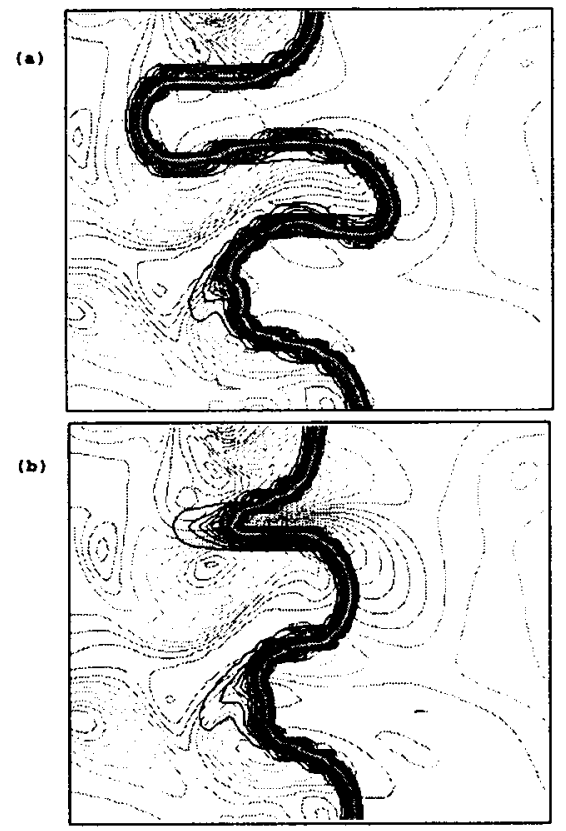

FIGURE 8. Superimposed instantaneous temperature and vorticity fields. Zoom from Fig. 6 Initial turbulence level $u_{0}^{\prime} / s_{l}^{0}=5$. (a) No imposed pressure gradient $\left(g^{*}=0\right.$. - case A); (b) Favorable imposed pressure gradient $\left(g^{*}=-6.25\right.$ - case B).
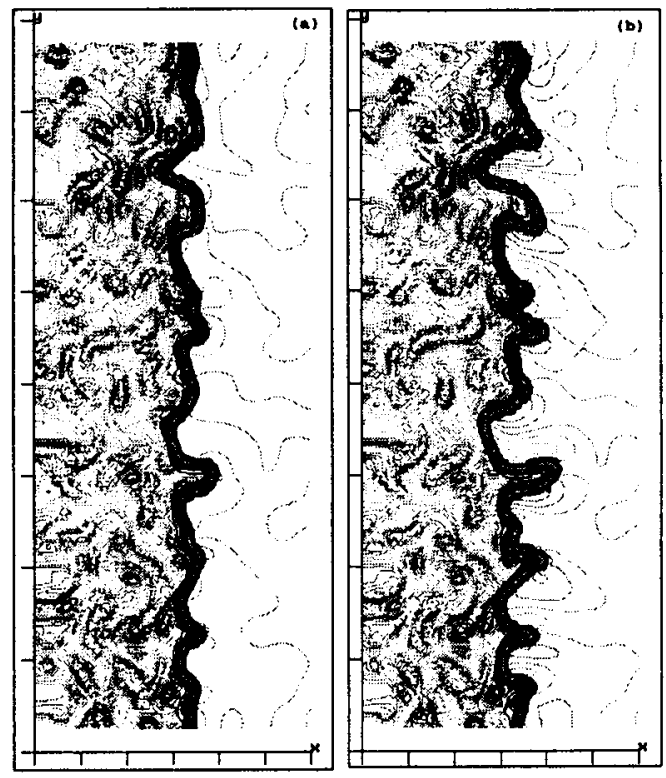

FIGURE 9. Superimposed instantaneous temperature and vorticity fields. Initial turbulence level $u_{0}^{\prime} / s_{l}^{0}=2$. (a) No imposed pressure gradient $\left(g^{*}=0\right.$. - case C); (b) Adverse imposed pressure gradient $\left(g^{*}=-6.25\right.$ - case $\left.\mathrm{E}\right)$. 

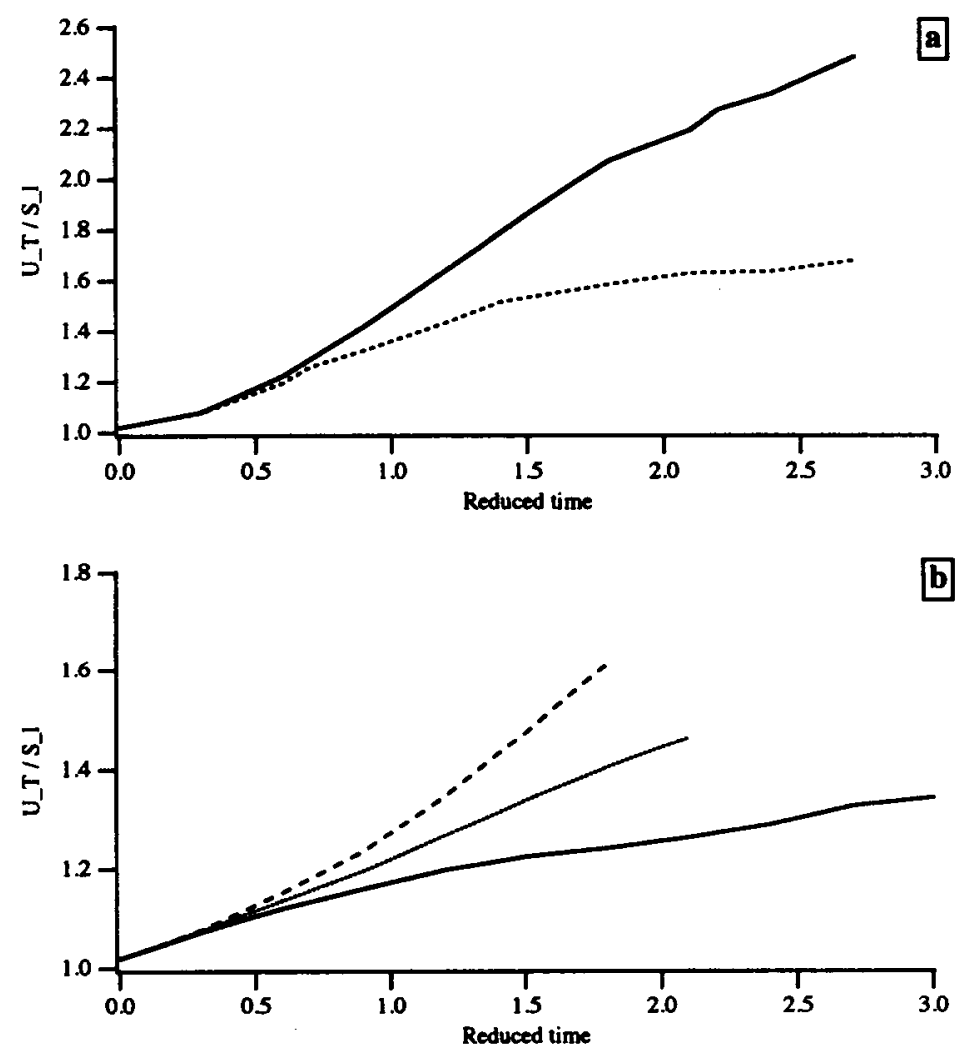

FIGURE 10. Reduced turbulent flame speed $S_{T} / s_{l}^{0}$ plotted as a function of the reduced time $t / t_{f}$, where $t_{f}=\delta_{l}^{0} / s_{l}^{0}$ is a flame time, for different values of $g^{*}$. (a) initial turbulent level $u_{0}^{\prime} / s_{1}^{0}=5$; cases A (-) and B (---- ); (b) initial turbulent level $u_{0}^{\prime} / s_{l}^{0}=2 ;$ cases $\mathrm{C}(-), \mathrm{D}(\cdots \cdots \cdots)$ ) and $\mathrm{E}(----)$.

Despite similar minimum and maximum values, the pressure field is mainly dominated by vortices in case $\mathrm{A}$ whereas the pressure gradient, imposed by the constant acceleration $\Gamma$, is clearly apparent for case B.

Close-ups of the temperature and vorticity fields of Fig. 6 are displayed in Fig. 8. As previously described, the flame front is less wrinkled in case $\mathrm{C}$ despite a similar turbulence distribution in the fresh gases. The internal flame front structure, however, is strongly modified by the mean pressure gradient and buoyancy effects. Protrusions of high temperature levels are clearly apparent in fresh gases. Accordingly, local flame front characteristics may be strongly affected, which will be investigated in the future.

Instantaneous temperature and vorticity fields are displayed for cases $\mathrm{C}$ and $\mathrm{E}$ in Fig. 9. The initial turbulence level is lower $\left(u_{0}^{\prime} / s_{l}^{0}=2\right)$ : without externally imposed pressure gradient, this flow exhibits a counter gradient turbulent transport as predicted by the Bray number criterion $\left(N_{B}=1.5\right)$. In case $\mathrm{E}$, an adverse pressure gradient is imposed and a transition towards gradient transport is expected. 

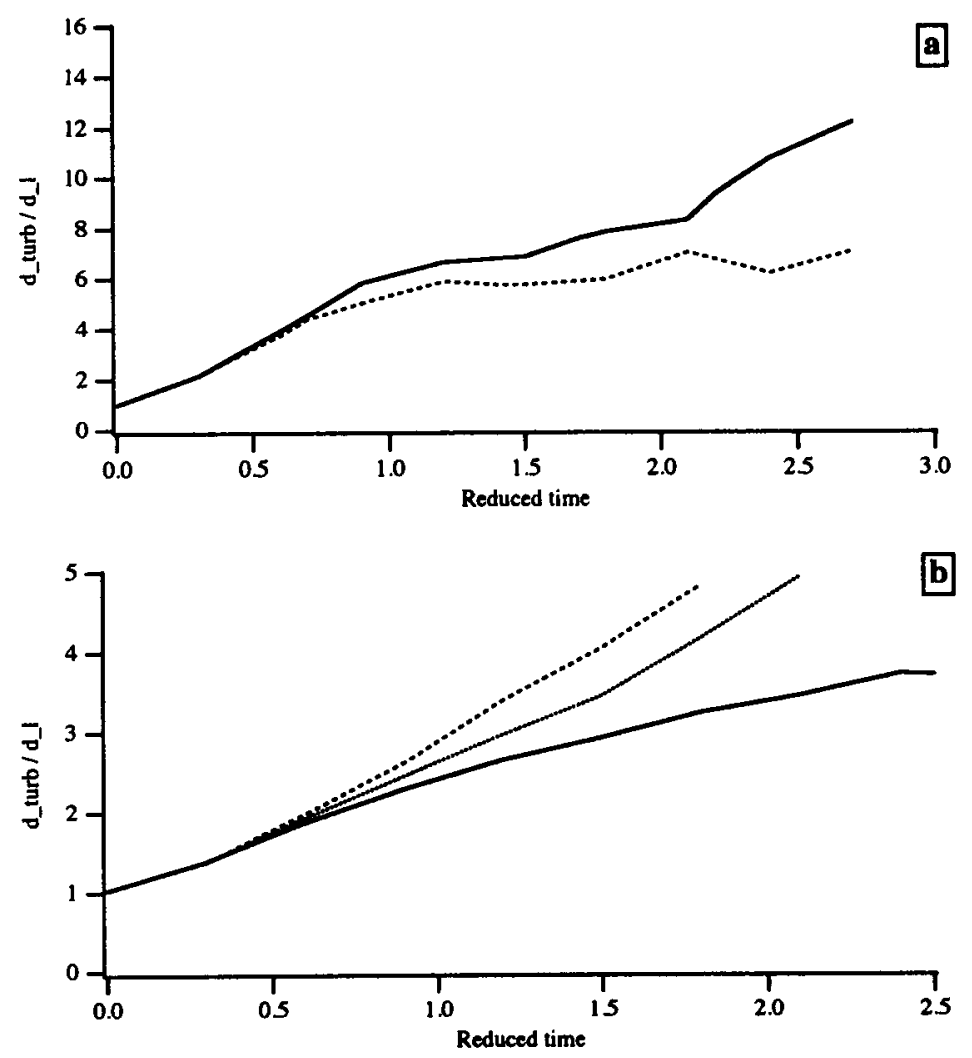

FIGURE 11. Reduced turbulent flame thickness $\delta_{T} / \delta_{l}^{0}$ plotted as a function of the reduced time $t / t_{f}$, where $t_{f}=\delta_{l}^{0} / s_{l}^{0}$ is a flame time, for different values of $g^{*}$. (a) initial turbulent level $u_{0}^{\prime} / s_{l}^{0}=5$; cases A (- ) and B (--- ); (b) initial turbulent level $u_{0}^{\prime} / s_{l}^{0}=2$; cases C (- $), \mathrm{D}(\cdots \cdots)$ ) and $\mathrm{E}(---)^{-}$. The turbulent flame brush is determined from the maximum value of the $\bar{c}$ gradient $\left(\delta_{t}=1 / \operatorname{Max}(\partial \bar{c} / \partial x)\right.$

The flame front wrinkling is somewhat increased by the adverse pressure gradient, due to the differential acceleration induced by buoyancy between fresh and burnt gases.

\subsection{Effect of the mean pressure gradient on global turbulent flame characteristics}

The global turbulent flame characteristics, namely the turbulent flame speed $S_{T}$ and flame brush thickness $\delta_{T}$, are plotted in Figs. 10 and 11 as a function of reduced time for different values of $g^{*}$. As expected from the previous flow-field visualizations, a favorable pressure gradient, i.e. $\partial \bar{P} / \partial x<0$, which is generally encountered in practical situations of confined turbulent flames, leads to a thinner turbulent flame brush and a lower turbulent flame speed. The decrease in $S_{T}$ may reach $30 \%$. On the other hand, an adverse pressure gradient, i.e. $\partial \bar{P} / \partial x>0$, induces an increase in flame brush thickness and a higher turbulent flame speed. These results are in agreement with the influence of a constant acceleration $\Gamma$ on 

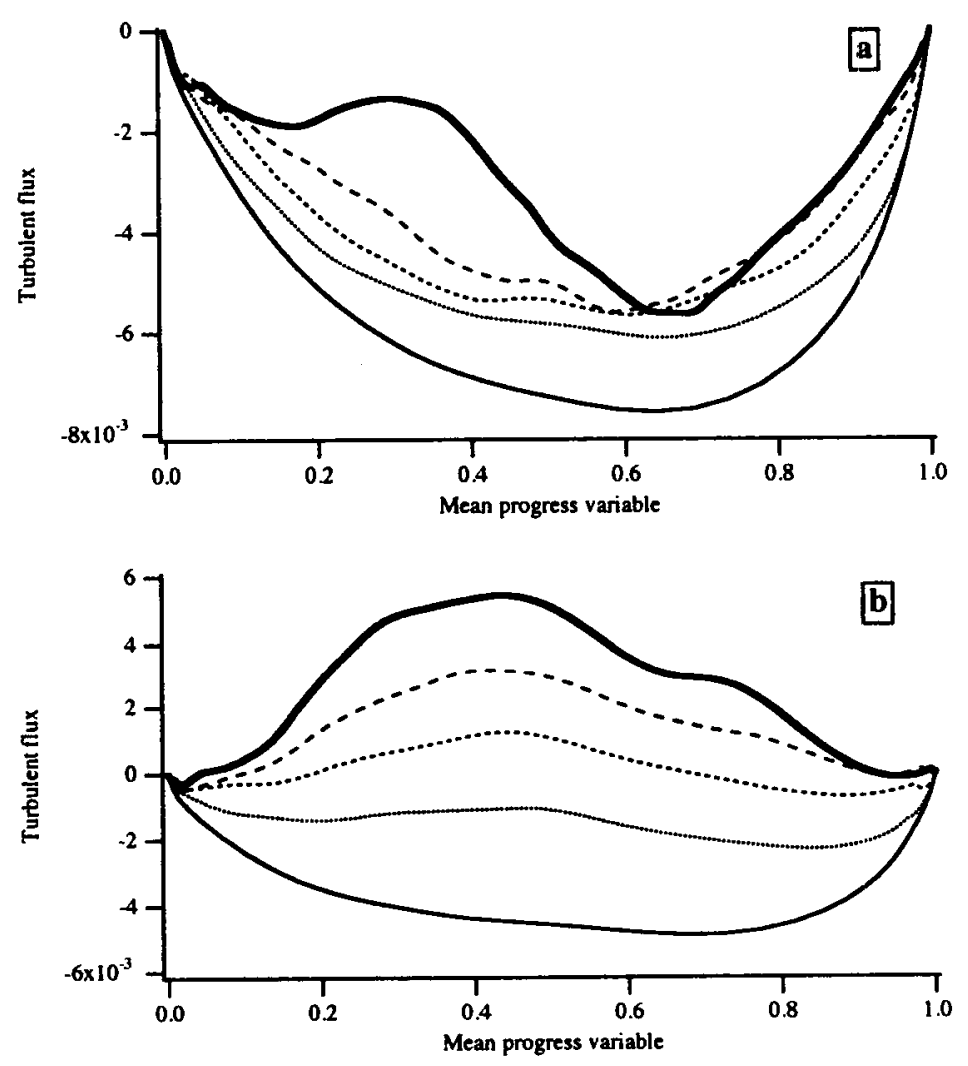

FIGURE 12. Transverse profiles of the turbulent flux $\bar{\rho} \widetilde{u_{1}^{\prime \prime} c^{\prime \prime}}$ plotted as a function of the mean progress variable $\widetilde{c}$ for different reduced time step $t^{+}=t / t_{f}$ where $t_{f}=\delta_{l}^{0} / s_{l}^{0}$ is a flame time. Initial turbulence level $u_{0}^{\prime} / s_{l}^{0}=5 . t^{+}=0.6(-) ; 0.9$ $(\cdots \cdots .$.$) ) 1.2(---) ; 1.5(---) ; 1.8(-)$. (a) No imposed pressure gradient $\left(g^{*}=0\right.$. - case A); (b) favorable imposed pressure gradient $\left(g^{*}=-6.25\right.$ - case B).

$S_{T}$ theoretically predicted by Libby (1989).

\subsection{Turbulent transport $\bar{\rho} \widetilde{u^{\prime \prime} c^{\prime \prime}}$}

The transverse profiles of the turbulent flux $\bar{\rho} \widetilde{u^{\prime \prime} c^{\prime \prime}}$ as a function of the mean progress variable $\tilde{c}$ for the cases $\mathrm{A}$ and $\mathrm{B}$ at various times are shown in Fig. 12. Case A, without an imposed mean pressure gradient, is clearly of gradient type, i.e. $\bar{\rho} \overline{u^{\prime \prime} c^{\prime \prime}}<0$, whereas the imposed favorable pressure gradient leads to a counter gradient turbulent transport. This finding is in agreement with the work of Bray et al.(1982) and is expected from Eq. (7). Even in clearly counter gradient situations, the turbulent flux $\bar{\rho} \widetilde{u^{\prime \prime} c^{\prime \prime}}$ is always negative, or of gradient type, at the leading edge of the flame brush, where $\tilde{c} \rightarrow 0$. As shown by Bray and his coworkers, these gradient zones allow flame stabilization.

The total turbulent flux, i.e. $\int_{-\infty}^{+\infty} \bar{\rho} \widetilde{u_{1}^{\prime \prime} c^{\prime \prime}} d x$, is plotted as a function of the reduced time for the different simulations in Fig. 13. Favorable pressure gradients promote 

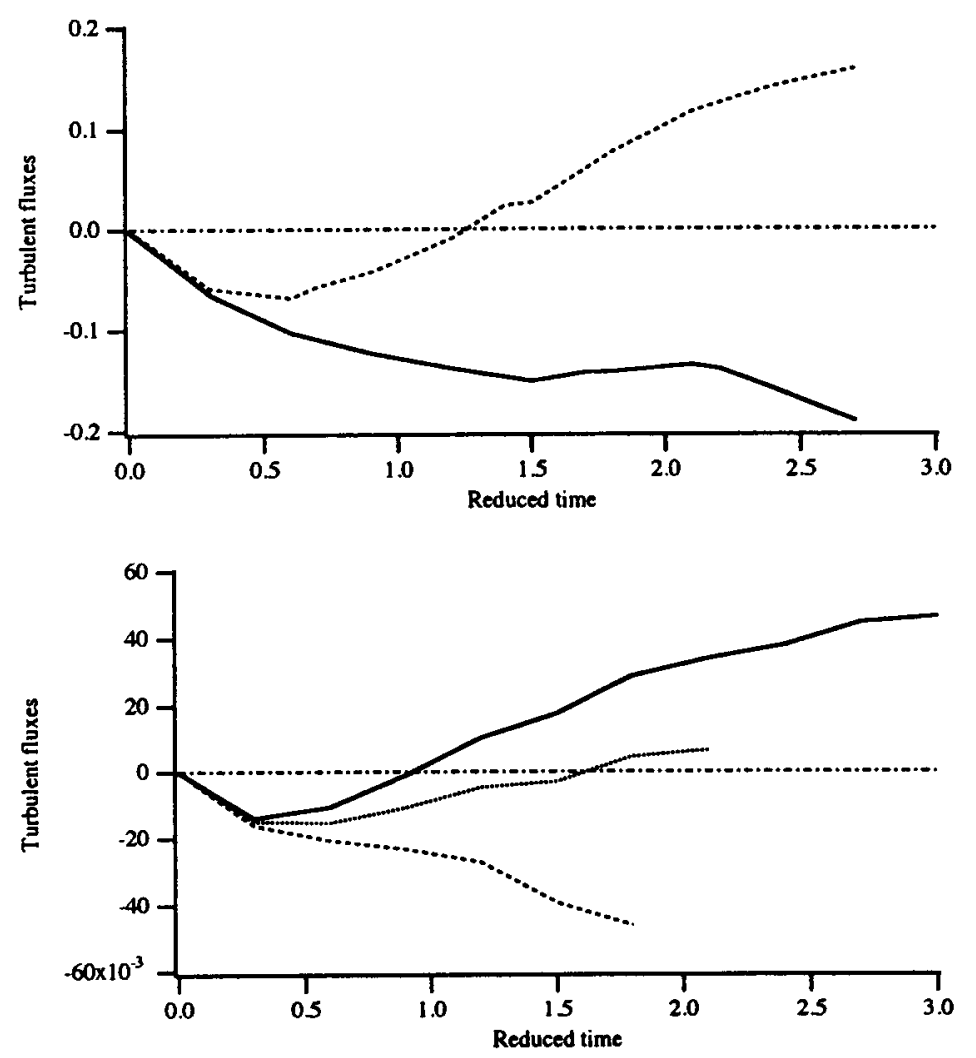

FIGURE 13. Total turbulent flux $\int_{-\infty}^{+\infty} \bar{\rho} \widetilde{u_{1}^{\prime \prime} c^{\prime \prime}} d x$ plotted as a function of the reduced time $t / t_{f}$ where $t_{f}=\delta_{l}^{0} / s_{l}^{0}$ is a flame time for the different cases. (a) initial turbulence level $u_{0}^{\prime} / s_{l}^{0}=5$ : Cases A (-) and B (---); (b) initial turbulence level $u_{0}^{\prime} / s_{l}^{0}=2$ : Cases $\mathrm{C}(-), \mathrm{D}(\cdots \cdots)$ ) and $\mathrm{E}(-\cdots)$.

counter-gradient diffusion, corresponding to a reduction of both the turbulent flame speed $S_{T}$ and the turbulent flame thickness $\delta_{T}$. On the other hand, adverse pressure gradients lead to an increase in $S_{T}$ and $\delta_{T}$ and induce gradient turbulent transport.

\subsection{Analysis of the $\bar{\rho} \widetilde{u^{\prime \prime} c^{\prime \prime}}$ transport equation}

All terms in Eq. 7 may be obtained from direct numerical simulations. This analysis serves to identify the dominant terms as well as the nature of their contribution. A typical direct numerical simulation evaluation of terms (I)-(X) appearing in the $\tilde{c}$-flux budget of Eq. 7 is presented in Fig. 14 for case $\mathrm{C}$. The figure also displays the imbalance that was found when numerically closing the $\tilde{c}$-flux budget in Eq. 7. This imbalance is due to inherent numerical errors involved in the simulations as well as in the post-processing of the data. Its magnitude remains small, which suggests that the simulations can be used to analyze the variations of second-order moments. For instance, Fig. 14 shows that the dissipation terms (VIII) and (IX), which are generally modeled together, are of the same order and act to promote gradient diffusion. 


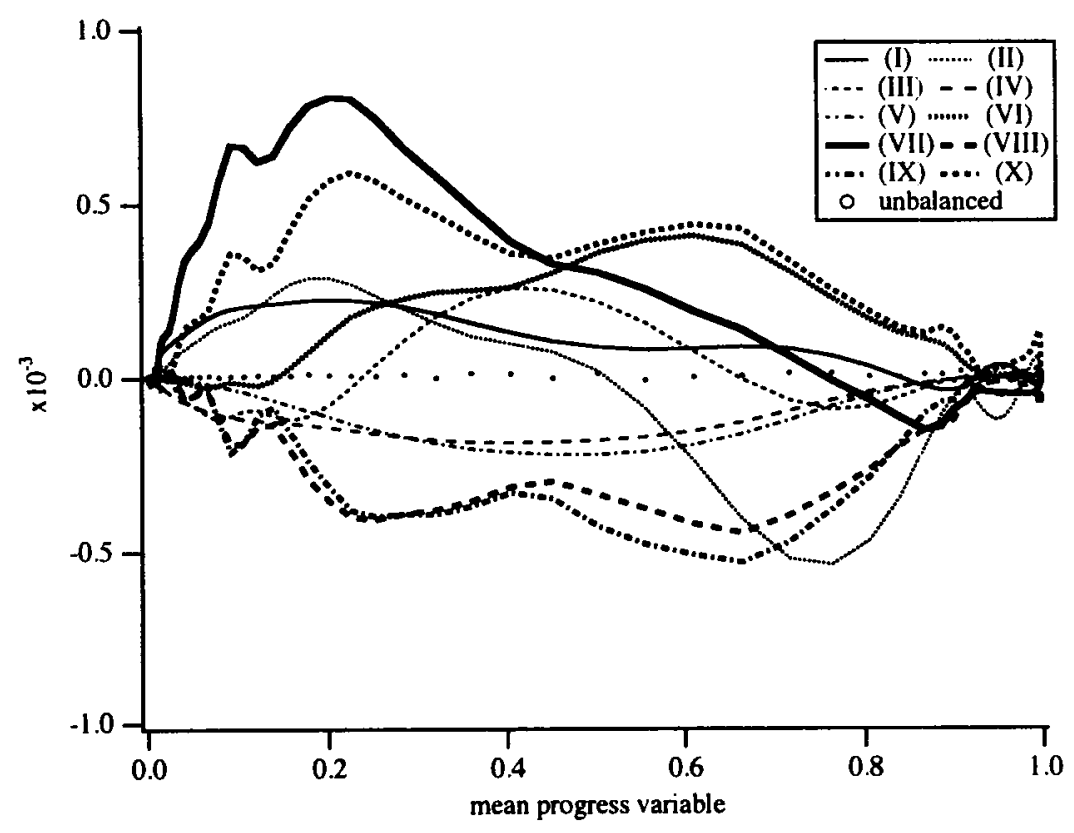

FiguRE 14. Variations of the different terms appearing in the $\tilde{c}$-flux budget across the turbulent flame brush. Case C: counter-gradient turbulent diffusion and no imposed pressure gradient.

On the other hand, pressure terms (VI) and (VII), and the velocity-reaction rate correlation $(\mathrm{X})$, strongly act to promote counter-gradient diffusion. The two source terms due to mean progress variable gradient (IV) and mean velocity gradient (V) tend to decrease the turbulent fluxes as expected and, accordingly, in the present counter-gradient situation act to promote gradient turbulent diffusion. The mean pressure gradient term corresponds to the pressure jump across the flame brush (see Fig. 17):

$$
-\overline{c^{\prime \prime}} \frac{\partial \bar{p}}{\partial x}=(\bar{c}-\widetilde{c}) \frac{\partial \bar{p}}{\partial x} \approx \tau \frac{\widetilde{c}(1-\widetilde{c})}{1+\tau \widetilde{c}} \frac{\rho_{u} \tau\left(s_{l}^{0}\right)^{2}}{\delta_{T}}
$$

The fluctuating pressure term (VII) cannot be neglected as generally assumed in the models proposed to close the transport equation (7).

A similar analysis is now performed for case $\mathrm{E}$, where, due to the imposed adverse pressure gradient, the turbulent diffusion becomes gradient type, as indicated in Fig. 15. As expected, the mean pressure gradient term tends to promote gradient turbulent diffusion and corresponds to the imposed pressure gradient. Once again, the fluctuating pressure term (VII) is not negligible and acts to counterbalance the mean pressure gradient term (VI). In fact, the combined term (VI) $+(\mathrm{VII})$ is mainly negative and corresponds to a gradient diffusion. The reaction term acts to promote counter-gradient diffusion.

The budget of the transport equation for $\bar{\rho} \widetilde{u_{1}^{\prime \prime} c^{\prime \prime}}$ for case B is presented in Fig. 16. 


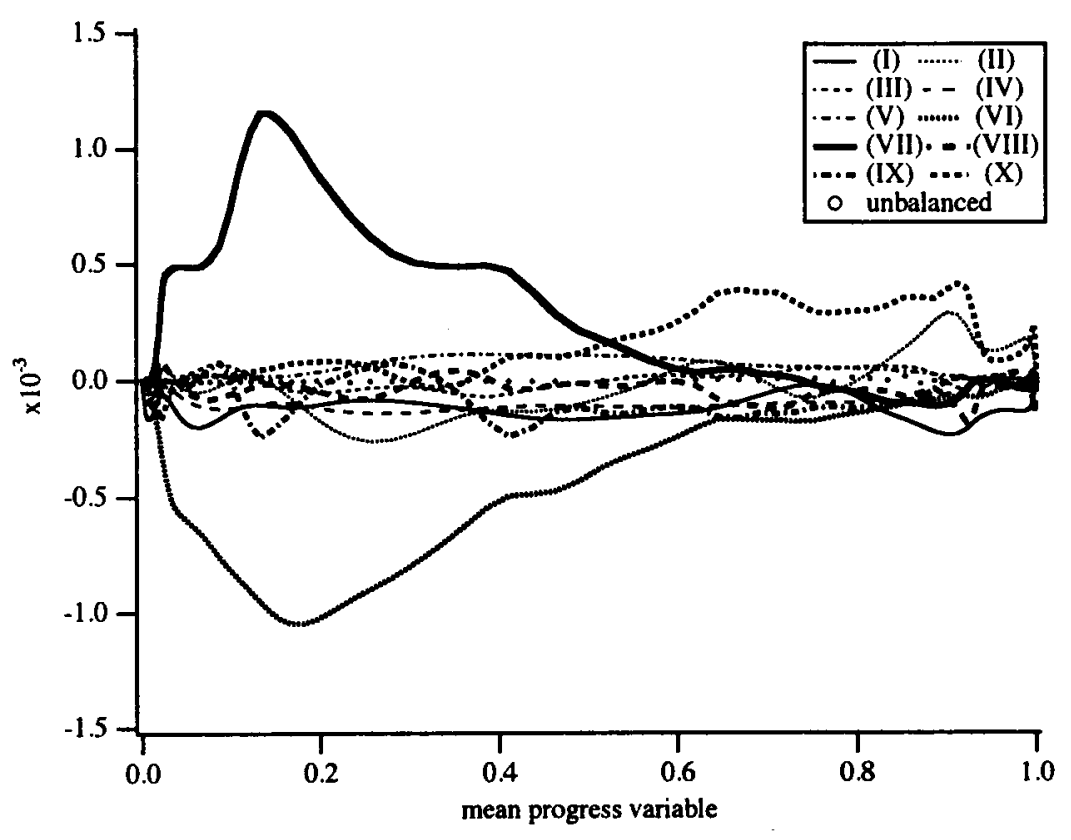

FIGURE 15. Variations of the different terms appearing in the $\tilde{c}$-flux budget across the turbulent flame brush. Case E: gradient turbulent diffusion induced by an adverse mean pressure gradient.

The favorable mean pressure gradient acts to promote counter-gradient turbulent diffusion from term (VI). Once again, term (VII) tends to counterbalance term (VI). For such a situation, Bray et al.(1982) propose to neglect the pressure fluctuation effects (term VII) and assume that only the cross-dissipation terms (VIII + IX) can provide a balance to the mean pressure term (VI). In fact, from our simulation, the mean pressure term (VI) is balanced by the sum of the three contributions: the cross-dissipation term (VIII + IX), the pressure fluctuation term (VII) that cannot be neglected, and the source term due to gradients of $\widetilde{c}$ (IV).

The mean pressure gradient across the flame brush may be simply modeled as the sum of two contributions: the imposed pressure gradient and the pressure jump due to thermal heat release. As a result, the source term (VI) in $\rho u^{\prime \prime} c^{\prime \prime}$ becomes:

$$
-\overline{c^{\prime \prime}} \frac{\partial \bar{p}}{\partial x}=(\bar{c}-\widetilde{c}) \frac{\partial \bar{p}}{\partial x} \approx \tau \frac{\widetilde{c}(1-\widetilde{c})}{1+\tau \tilde{c}}\left[\frac{\rho_{u} \tau\left(s_{l}^{0}\right)^{2}}{\delta_{T}}-\bar{\rho} \Gamma\right]
$$

where $\rho \Gamma$ corresponds to the imposed pressure gradient. This expression is verified in the present simulations (Fig. 17).

\section{Theoretical analysis and modeling}

\subsection{Model for turbulent fiux without pressure gradient}

In this section, we first recall the derivation of a model for the turbulent flux of the mean progress variable $\tilde{c}$. Details about this modeling may be found in Veynante $e t$ 


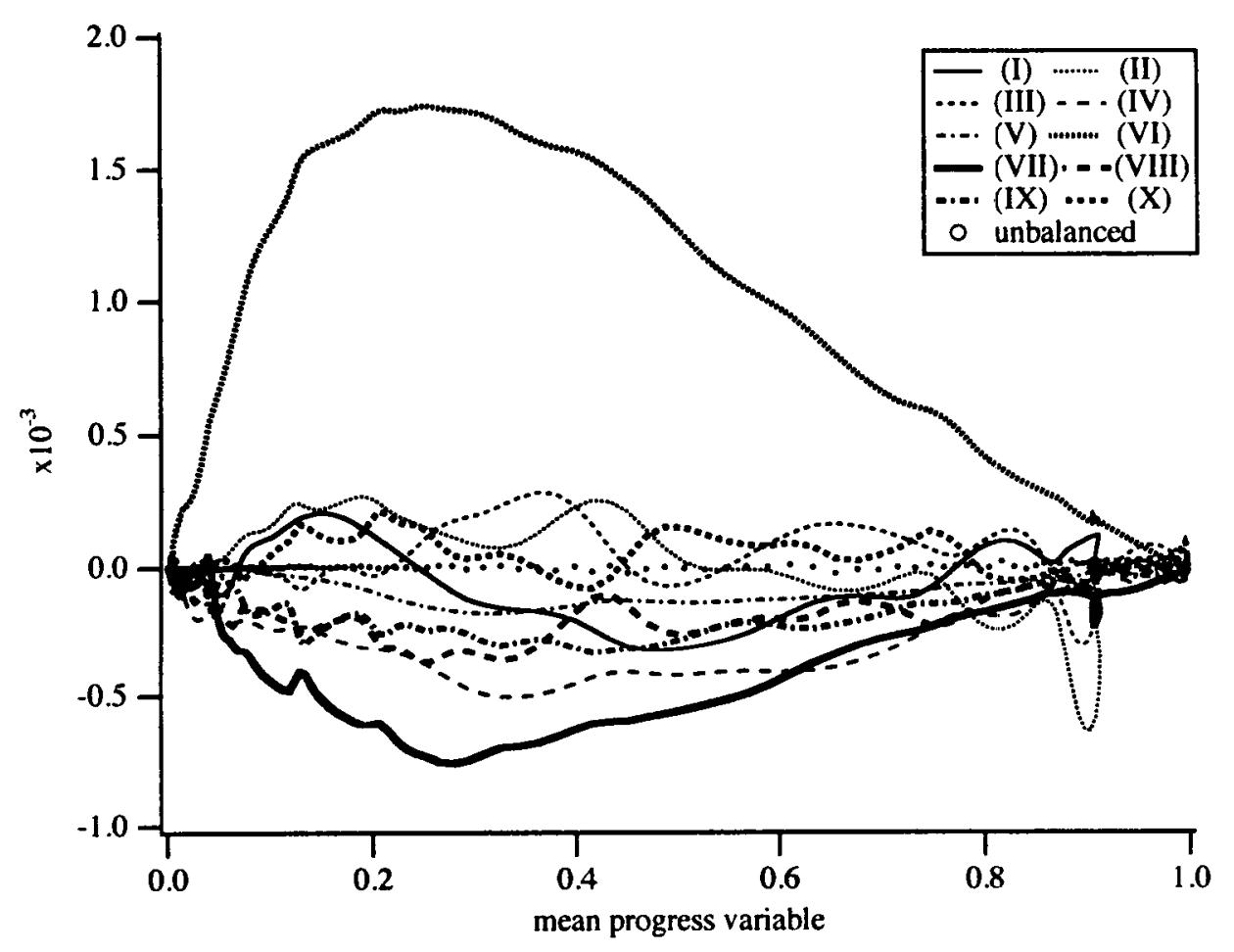

FIgURE 16. Variations of the different terms appearing in the $\widetilde{c}$-flux budget across the turbulent flame brush. Case B: counter-gradient turbulent diffusion induced by a favorable mean pressure gradient.

al.(1995). This derivation starts from a relation proposed by Bidaux \& Bray (1994) connecting the flame front averaged fluctuating velocity, $\left\langle u_{i}^{\prime \prime}\right\rangle_{s}$, to the conditioned unburnt and burnt mean velocities:

$$
\left\langle u_{i}^{\prime \prime}\right\rangle_{s}=\left(c^{*}-\tilde{c}\right)\left(\overline{u_{i b}}-\overline{u_{i u}}\right)
$$

where $c^{*}$ corresponds to the iso- $c$ line used to define the flame location.

From Eq. (4), the previous relation leads to:

$$
\left\langle u_{i}^{\prime \prime}\right\rangle_{s}=\frac{\left(c^{*}-\tilde{c}\right)}{\widetilde{c}(1-\tilde{c})} \widetilde{u_{i}^{\prime \prime} c^{\prime \prime}}
$$

This expression may be used to derive an estimate of $\widetilde{u_{i}^{\prime \prime} c^{\prime \prime}}$ via a model for the mean velocity fluctuation $\left\langle u_{i}^{\prime \prime}\right\rangle_{s}$; considering limiting cases of low turbulence levels where flow dynamic is mainly controlled by the thermal expansion across the flame brush, and high turbulence levels where the turbulent velocities dominate the flow induced by thermal expansion, Veynante et al.(1995) proposed the following expression for $\left\langle u_{1}^{\prime \prime}\right\rangle_{\text {s }}$ where index 1 corresponds to the direction normal to the flame:

$$
\left\langle u_{1}^{\prime \prime}\right\rangle_{s}=\left(c^{*}-\tilde{c}\right)\left(\tau s_{L}-2 \alpha u^{\prime}\right)
$$




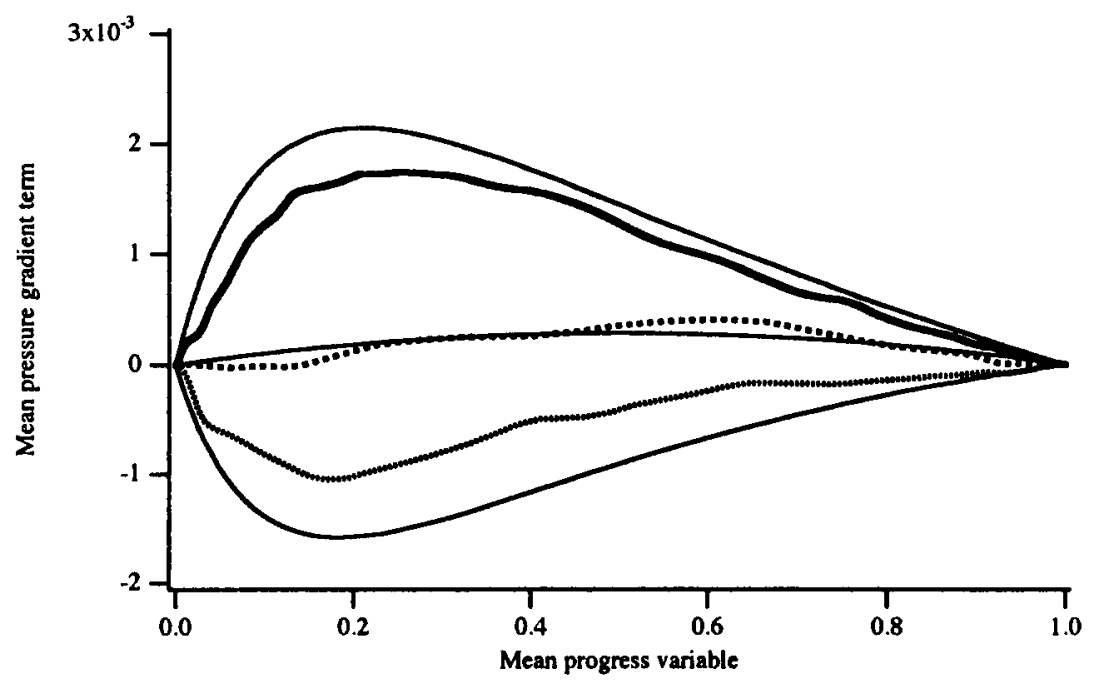

Figure 17. Comparison between direct numerical simulation data for cases B $(-), \mathrm{C}(---)$ and $\mathrm{E}(\cdots \cdots . .$.$) and modeled mean pressure gradient source$ term ( $(-)$ from Eq. (23) in the the $\tilde{c}$-flux transport equation (Eq. 7).

leading to:

$$
\widetilde{u_{1}^{\prime \prime} c^{\prime \prime}}=\widetilde{c}(1-\tilde{c})\left(\tau s_{L}-2 \alpha u^{\prime}\right)
$$

and to the Bray number criterion. Here $u^{\prime}$ denotes the rms velocity fluctuations and $\alpha$ is an efficiency function to take into account the low ability of small turbulent vortices to affect the flame front.

\subsection{Model for turbulent flux with pressure gradient}

Our objective in this section is to incorporate pressure gradient effects in the previous analysis. Pressure gradients induce differential buoyancy effects between cold heavy reactants and light hot products. We quantify this buoyancy effect through a characteristic velocity $U_{B}(\widetilde{c})$, which is simply added to the two velocities used in Eq. (26): the velocity induced by the flame, $\left(c^{*}-\widetilde{c}\right) \tau s_{L}$; and the velocity induced by turbulence, $2\left(c^{*}-\widetilde{c}\right) \alpha u^{\prime}$,

$$
\left\langle u_{1}^{\prime \prime}\right\rangle_{s}=\left(c^{*}-\widetilde{c}\right)\left(\tau s_{L}-2 \alpha u^{\prime}\right)+U_{B}(\widetilde{c})
$$

The estimation of $U_{B}(\widetilde{c})$ is done as follows. In the fresh gases, $U_{B}(0)=U_{B}^{b}$ corresponds to the relative speed of a pocket of burnt gas (density $\rho_{b}$, diameter $l$ ). 
Similarly a velocity $U_{B}(1)=U_{B}^{u}$ will be associated to the movement of unburnt gas pockets in the burnt products. Assuming an equilibrium between buoyancy and drag forces and using Stokes law for the drag of the pocket, $U_{B}^{b}$ is given by:

$$
U_{B}^{b} \approx-\frac{1}{12} \frac{\tau}{\tau+1} \frac{l^{2}}{\nu_{u}} \Gamma
$$

where $\nu_{u}$ is the kinematic viscosity in the fresh gases.

For a pocket of fresh gases with density $\rho_{u}$ and diameter $l$, the same analysis leads to a relative displacement speed $U_{B}^{u}$ :

$$
U_{B}^{u} \approx \frac{1}{12} \frac{\tau l^{2}}{\nu_{b}} \Gamma
$$

where $\nu_{b}$ is the kinematic viscosity in the burnt gases.

Assuming a linear variation of the buoyancy velocity $U_{B}(\tilde{c})$ with $\tilde{c}$ between the flame leading and trailing edges leads to:

$$
U_{B}(\tilde{c}) \approx \frac{1}{12} \frac{\tau}{\tau+1} \frac{l^{2}}{\nu_{u}} \Gamma\left[\left(1+(\tau+1)^{1-n}\right) \tilde{c}-1\right]
$$

where the kinematic viscosity ratio has been estimated as: $\frac{\nu_{u}}{\nu_{b}} \approx\left(\frac{T_{u}}{T_{b}}\right)^{n}=\left(\frac{1}{\tau+1}\right)^{n}$ and $n=1.76$.

For $3 \leq \tau \leq 6$, we have $0.74 \leq 1 /\left(1+(\tau+1)^{1-n}\right) \leq 0.8$. In a first step, to achieve simple expressions, the c-level retained to define the flame front may be chosen as $c^{*}=1 /\left(1+(\tau+1)^{1-n}\right)$, leading to:

$$
U_{B}(\tilde{c}) \approx \frac{1}{12} \frac{\tau}{c^{*}(\tau+1)} \frac{l^{2}}{\nu_{u}} \Gamma\left(\widetilde{c}-c^{*}\right)
$$

Then, from Eq. (25) and (28), a simple model for the turbulent flux $\widetilde{u_{1}^{\prime \prime} c^{\prime \prime}}$ is:

$$
\widetilde{u_{1}^{\prime \prime} c^{\prime \prime}}=\widetilde{c}(1-\widetilde{c})\left(\tau s_{L}-2 \alpha u^{\prime}-\beta \frac{\tau l^{2}}{12 c^{*}(\tau+1) \nu_{u}} \Gamma\right)
$$

where a model constant $\beta$ is introduced to take into account the various limitations of the simplified analysis proposed here.

The previous expression needs a characteristic length $l$ corresponding to the typical size of a pocket of fresh gases in a medium of burnt gases, and vice versa. As a first step, the integral length scale $l_{t}$, a rough estimate of the flame front wrinkling scale, is used. Predictions from expression (33) are compared with simulation data for the reduced time 1.8 in Fig. 18 using $c^{*}=0.8$ and $\beta=0.12$. The efficiency function, $\alpha$, is a function of the length scale ratio $l_{t} / \delta_{l}$ and is obtained from previous direct numerical simulations (Veynante et al., 1995) to be about 0.5 for the length 


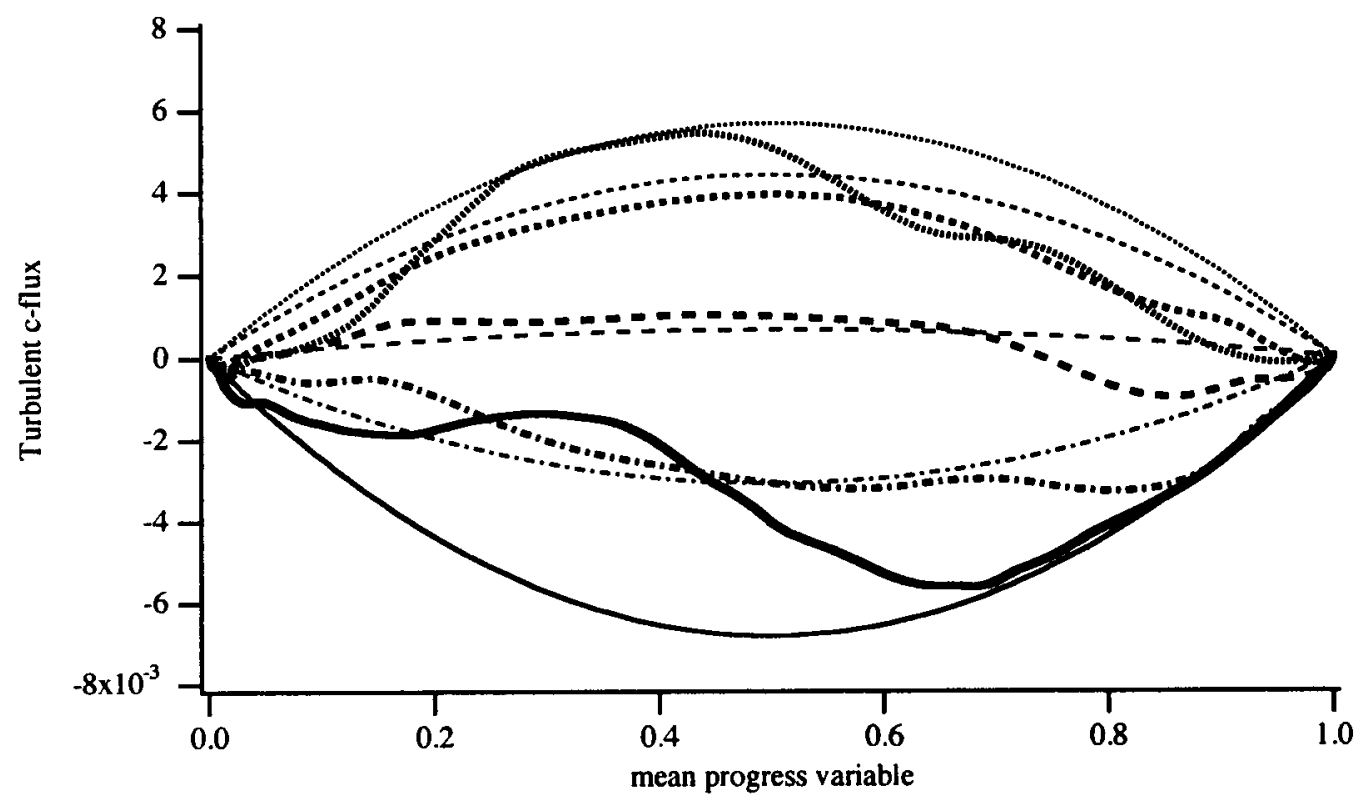

FIGURE 18. Comparison between direct numerical simulation data (Bold curves) and model predictions (thin curves) from Eq. 33 of the turbulent flux $\widetilde{u_{1}^{\prime \prime} c^{\prime \prime}}$ as a function of the mean progress variable $\tilde{c}$ at reduced time 1.8 for cases $\mathrm{A}(-)$, $\mathrm{B}(\cdots \cdots),. \mathrm{C}(-\cdots), \mathrm{D}(---)$, and $\mathrm{E}(-\cdots), \alpha=0.5, \beta=0.12$ and $c^{*}=0.8$. The characteristic length $l$ is taken equal to the integral length scale $l_{t}$

scale ratio used here. The agreement between numerical data and model predictions is satisfactory. The influence of the imposed acceleration and the transition between gradient and counter-gradient transport are well predicted from Eq. (33).

A simple criterion may be derived to predict the occurrence of counter-gradient turbulent diffusion, i.e. $\widetilde{u_{1}^{\prime \prime} c^{\prime \prime}} \geq 0$ ), from Eq. 33:

$$
N_{B}^{\Gamma}=\frac{\tau}{2 \alpha \frac{u^{\prime}}{s_{L}}}\left[1-\beta \frac{l^{2} \Gamma}{12 c^{*}(\tau+1) \nu_{u} s_{L}}\right] \geq 1
$$

The effect of the constant acceleration is to introduce a coefficient for the Bray number $N_{B}$ defined by Veynante et al.(1995), see Eq. (3). $N_{B}^{\Gamma}$ may be rewritten as:

$$
N_{B}^{\Gamma}=\frac{\tau}{2 \alpha \frac{u^{\prime}}{s_{L}}}\left[1-\beta \frac{g^{*} R e_{f}}{12 c^{*}(\tau+1)}\left(\frac{l}{\delta_{l}}\right)^{2}\right] \geq 1
$$

where $R e_{f}=\delta_{l} s_{L} / \nu_{u}$ is a flame Reynolds number. 
This modified Bray number may be estimated from our numerical simulation using the initial turbulence values $\left(l=l_{t}, u^{\prime}=u_{0}^{\prime}, s_{L}=s_{l}^{0}\right), \alpha=0.5$ (from (Veynante et al., 1995), $\beta=0.12$ and $c^{*}=0.8$. Results are summarized and validated in Table IV; it appears that $N_{B}^{\Gamma} \geq 1$ flows indeed exhibit counter-gradient diffusion.

The same analysis may be easily extended to the case of an externally imposed pressure gradient $\partial \bar{P} / \partial x$, leading to

$$
U_{B}(\widetilde{c}) \approx \frac{\tau l^{2}}{12 \rho_{u} \nu_{u}}\left(\frac{\partial \bar{P}}{\partial x}\right)\left[\left(1+(\tau+1)^{1-n}\right) \tilde{c}-1\right]
$$

and

$$
\widetilde{u_{1}^{\prime \prime} c^{\prime \prime}}=\widetilde{c}(1-\widetilde{c})\left(\tau s_{L}-2 \alpha u^{\prime}-\beta \frac{\tau l^{2}}{12 \rho_{u} \nu_{u} c^{*}}\left(\frac{\partial \bar{P}}{\partial x}\right)\right)
$$

This expression leads to the following criterion for counter-gradient turbulent transport:

$$
\begin{gathered}
N_{B}^{p}=\frac{\tau}{2 \alpha \frac{u^{\prime}}{s_{L}}}\left[1-\beta \frac{\tau \nabla p^{*} R e_{f}}{12 c^{*}}\left(\frac{l}{\delta_{l}}\right)^{2}\right] \geq 1 \\
\nabla p^{*}=\left(\frac{\partial \bar{P}}{\partial x}\right) \frac{\delta_{l}}{\rho_{u} \tau s_{l}^{2}}
\end{gathered}
$$

which is the reduced pressure gradient (i.e. the pressure gradient made nondimensionalized by the pressure gradient across the corresponding laminar flame). The previous result is based on a simple analysis, assuming an equilibrium between buoyancy and drag forces for a pocket of fresh (burnt) gases in a medium of burnt (fresh) gases. This approach is probably too crude and has to be improved to describe the instability mechanisms of an interface between two gases with different densities. The dependence of $\widetilde{u^{\prime \prime} c^{\prime \prime}}$ with the square of the integral length scale $l_{t}$ needs to be examined and validated, especially for large values of $l_{t}$.

TABLE IV. Estimation of $N_{B}^{\Gamma}$. GD (CGD) refers to gradient (counter-gradient) turbulent diffusion

\begin{tabular}{llllll}
\hline Case & $u_{0}^{\prime} / s_{l}^{0}$ & $l_{t} / \delta_{l}^{0}$ & $g^{*}$ & GD/CGD & $N_{B}^{\Gamma}$ \\
\hline $\mathrm{A}$ & 5. & 3.5 & 0. & GD & 0.60 \\
$\mathrm{~B}$ & 5. & 3.5 & -6.25 & CGD & 1.3 \\
$\mathrm{C}$ & 2. & 2.7 & 0. & CGD & 1.5 \\
$\mathrm{D}$ & 2. & 2.7 & 3.12 & GD & 0.96 \\
$\mathrm{E}$ & 2. & 2.7 & 6.25 & GD & 0.43
\end{tabular}

In the experiment of Shepherd et al.(1982), $u^{\prime} \approx 1 \mathrm{~m} / \mathrm{s}$ and $l_{t} \approx 1 \mathrm{~cm}$, leading to Bray numbers $N_{B} \approx 0.9$ and $N_{B}^{p} \approx 20$. Due to the large length scale ratio, 
$l_{t} / \delta_{l} \approx 20, \alpha=1$, (cf. Veynante et al.1995). These results are compared in Table $\mathrm{V}$ with the experimental estimation of the turbulent flux $\bar{\rho} \widetilde{u_{1}^{\prime \prime} c^{\prime \prime}}$. Accordingly, the unconfined case without pressure gradients lies in the transition region between gradient and counter-gradient diffusion. In fact, counter-gradient diffusion is found in the experiment, but the turbulent flux remains quite low. On the other hand, the confined case with an externally imposed pressure gradient, $N_{B}^{p}$ measures the intensity and the sign of the turbulent flux $\rho \widetilde{u_{1}^{\prime \prime} c^{\prime \prime}}$. The large value of $N_{B}^{p}$ for the confined experiment is questionable because of the large length scale ratio $\left(l_{t} / \delta_{l}\right)$.

TABLE V. Estimation of $N_{B}^{p}$ in the Shepherd et al.(1982) experiment for the confined (with pressure gradients) and unconfined (without pressure pressure gradients). Experimental estimations of $\bar{\rho} \widetilde{u}_{1}^{\prime \prime} c^{\prime \prime} / \bar{\rho} \widetilde{U}_{0}$, where $\widetilde{U}_{0}$ is the reference burner inlet velocity, are provided. $u^{t} \approx 1 \mathrm{~m} / \mathrm{s}, l_{t} \approx 1 \mathrm{~cm}$.

\begin{tabular}{lll}
\hline Case & $N_{B}^{p}$ & $\bar{\rho} \widetilde{u_{1}^{\prime \prime} c^{\prime \prime}} / \bar{\rho} \widetilde{U}_{0}$ \\
\hline unconfined & 0.9 & 0.0043 \\
confined & 20. & 0.051 \\
\hline
\end{tabular}

\section{Conclusion}

The influence of a constant acceleration $\Gamma$ on a turbulent premixed flame is studied by direct numerical simulation. This acceleration $\Gamma$ induces a mean pressure gradient across the flame brush, leading to a modification of the turbulent flame structure due to differential buoyancy mechanisms between heavy cold fresh and light hot burnt gases. Such a pressure gradient may be encountered in practical applications in ducted flames.

A favorable pressure gradient, i.e. the pressure decreases from unburnt to burnt gases, is found to decrease the flame wrinkling, the flame brush thickness, and the turbulent flame speed. A favorable pressure gradient also promotes counter-gradient turbulent transport. On the other hand, adverse pressure gradients tend to increase the flame brush thickness and turbulent flame speed, and promote classical gradient turbulent transport.

The balance equation for the turbulent flux $\bar{\rho} \widetilde{u^{\prime \prime} c^{\prime \prime}}$ of the Favre averaged progress variable $\bar{c}$ is also analyzed. The first results show that the fluctuating pressure term, $\left(\overline{c^{\prime \prime} \partial p^{\prime} \partial x}\right)$, cannot be neglected as generally assumed in models. Simple models assuming that a high mean pressure gradient may only be balanced by the cross-dissipation term seem too approximate. This analysis has to be continued to compare simulation data and closure schemes proposed for the $\bar{\rho} u^{\prime \prime} c^{\prime \prime}$ transport equation.

The analysis developed by Veynante et al.(1995) has been extended to imposed acceleration and mean pressure gradients. A simple model for the turbulent flux $\widetilde{u^{\prime \prime} c^{\prime \prime}}$ is proposed and validated from simulation data. Then, a modified criterion is derived to delineate between counter-gradient and gradient turbulent diffusion. In 
fact, counter-gradient diffusion may occur in most practical applications, especially for ducted flames.

\section{REFERENCES}

Bray, K. N. C. 1980 Turbulent flows with premixed reactants. in Topics in Applied Physics 44. Springer-Verlag.

Bray, K. N. C., Libby, P. A., Masuya, G. \& Moss, J. B. 1981 Turbulence production in premixed turbulent flames. Comb. Sci. Tech. 25, 127-140.

Bray, K. N. C., Moss, J. B. \& LibBy, P. A. 1982 Turbulent transport in premixed flames in Convective Transport and Instability Phenomena, J. Zierep and H. Oertel, University of Karlsruhe.

Bray, K. N. C., Champion, M. \& LibBy, P. A. 1989 The interaction between turbulence and chemistry in premixed turbulent flames. In Turbulent Reactive Flows, Lecture Notes in Eng. 40, Springer-Verlag.

Bray, K. N. C. 1990 Studies of the turbulent burning velocity. Proc. R. Soc. Lond. A. 431, 315-335.

HaworTh, D. C. \& PoINSOT, T. 1992 Numerical simulations of Lewis number effects in turbulent premixed flames. J. Fluid Mech. 244, 405-436.

LiBBY, P. A. \& BrAY, K. N. C. 1981 Countergradient diffusion in premixed turbulent flames. AIAA J. 19, 205-213.

LIBBY, P. A. 1989 Theoretical analysis of the effect of gravity on premixed turbulent flames. Comb. Sci. Tech. 68, 15-33.

MASUYA, G. \& LIBBY, P. A. 1981 Nongradient theory for oblique turbulent flames with premixed reactants. A.I.A.A. Journal. 19, 1590-1599.

Moss J. B. 1980 Simultaneous Measurements of Concentration and Velocity in an Open Premixed Turbulent Flame. Combust. Sci. Tech. 22, 119-129.

PoinsoT, T, \& LELE, S. K. 1992 Boundary conditions for direct numerical simulations of compressible viscous flows. J. Comp. Phys. 101, 104-129.

Rutland, C. J. \& CANT, R. S. 1994 Turbulent transport in premixed flames. Proc. of the Summer Program, Center for Turbulence Research, NASA Ames/Stanford Univ.

Shepherd, I. G., Moss, J. B. \& Bray, K. N. C. 1982 Turbulent transport in a confined premixed flame. Nineteenth Symp. (International) on Combust. 423-431. The Combustion Institute.

Trouvé, A., Veynante, D. Bray, K. N. C. \& Mantel, T. 1994 The coupling between flame surface dynamics and species mass conservation in premixed turbulent combustion. Proc. of the Summer Program, Center for Turbulence Research, NASA Ames/Stanford Univ. 
Veynante, D., Trouvé, A., Bray, K. N. C. \& Mantel T. 1995 Gradient and counter-gradient scalar transport in turbulent premixed flames. (Submitted to J. Fluid Mech.)

Williams, F. A. 1985 Combustion theory. 2nd ed., Benjamin Cummings.

\section{Appendix I: tests of NTMIX with constant force or accelerations}

To validate the implementation of volume forces in the simulation, simple examples of one-dimensional flows with constant force or acceleration have been run. We consider an isentropic one-dimensional flow submitted to a force $F_{v} . F_{v}$ is either constant or equal to $\rho \gamma$. The governing equations are:

$$
\begin{gathered}
\frac{\partial(\rho u)}{\partial x}=0 \\
\frac{\partial\left(\rho u^{2}+p\right)}{\partial x}=F
\end{gathered}
$$

For constant volume force $F_{v}, F$ is constant and equal to $F_{v}$ and for constant acceleration, $F=\rho \Gamma$.

This system is integrated once to give:

$$
\begin{gathered}
\rho u=\rho_{0} u_{0} \\
\frac{\partial u}{\partial x}=-\frac{u_{0}}{c_{0}^{2} \rho_{0}} \cdot \frac{F}{\left(u_{0} / u\right)^{\gamma+1}\left(1 .-M^{2}\left(u / u_{0}\right)^{\gamma+1}\right)}
\end{gathered}
$$

where index 0 designates the inlet condition. $M=u / c$ is the local Mach number and $\gamma=1.4$.

If the local Mach number $M$ is small, this system may be integrated easily to give:

For constant volume force $F_{v}$ :

$$
\begin{gathered}
u(x)=u_{0}\left(1-\frac{F_{v} x}{P_{0}}\right)^{-1 / \gamma} \\
\rho(x)=\rho_{0}\left(1-\frac{F_{v} x}{P_{0}}\right)^{1 / \gamma} \\
P(x)=P_{0}-F_{v} x
\end{gathered}
$$

Note that $\frac{\partial P}{\partial x}=-F_{v}$

For constant acceleration $\Gamma$ :

$$
\begin{gathered}
u(x)=u_{0}\left(1+\frac{(\gamma+1) \Gamma x}{c_{0}^{2}}\right)^{-1 /(\gamma+1)} \\
\rho(x)=\rho_{0}\left(1+\frac{(\gamma+1) \Gamma x}{c_{0}^{2}}\right)^{1 /(\gamma+1)}
\end{gathered}
$$




$$
P(x)=P_{0}\left(1+\frac{(\gamma+1) \Gamma x}{c_{0}^{2}}\right)^{\gamma /(\gamma+1)}
$$

where $c_{0}$ is the inlet sound speed $\left(c_{0}=\gamma P_{0} / \rho_{0}\right)$.

For small values of $\Gamma, \frac{\partial P}{\partial x}=\rho_{0} \Gamma$

Fig. 19 shows (for a constant volume force $F_{v} / \rho_{0} c_{0}^{2}=0.01$ ) that the simulation results match these analytical expressions well. (A similar agreement is obtained in the case of a constant acceleration)

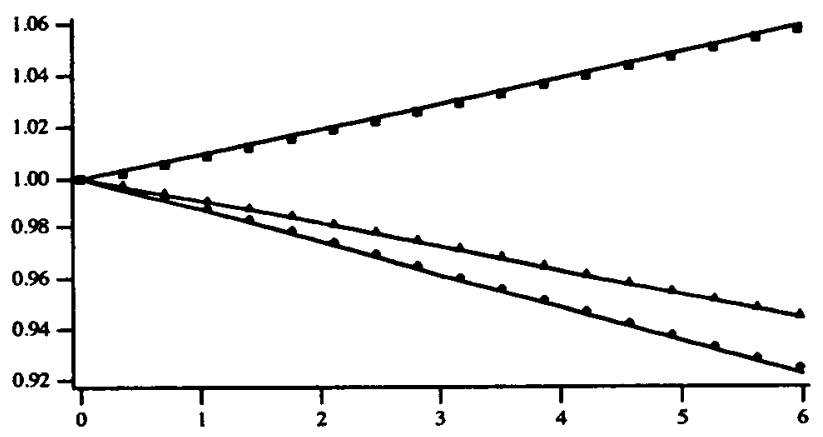

FIgURE 19. Tests of code for one-dimensional flow with constant volume force $F_{v} / \rho_{0} c_{0}^{2}=0.01$. Comparison between simulation data: velocity $(\bullet)$, pressure $(\diamond)$, density $(\bullet)$ and analytical results $(-\infty)$. 